\title{
An observational survey of molecular emission ahead of Herbig-Haro objects ${ }^{\star}$
}

\author{
S. Viti ${ }^{1}$, J. M. Girart ${ }^{2,3}$, and J. Hatchell ${ }^{4}$ \\ 1 Department of Physics and Astronomy, UCL, Gower St., London, WC1E 6BT, UK \\ e-mail: sv@star.ucl.ac.uk \\ 2 Institut de Ciències de l'Espai (CSIC), Gran Capità 2, 08034 Barcelona, Catalunya, Spain \\ 3 Institut d'Estudis Espacial de Catalunya, Spain \\ ${ }^{4}$ School of Physics, University of Exeter, Stocker Road, Exeter, EX4 4QL, UK
}

Received 20 October 2005 / Accepted 16 December 2005

\section{ABSTRACT}

Context. A molecular survey recently performed ahead of HH 2 supports the idea that the observed molecular enhancement is due to UV radiation from the $\mathrm{HH}$ object.

Aims. The aim of the present work is to determine whether all $\mathrm{HH}$ objects with enhanced $\mathrm{HCO}^{+}$emission ahead of them also exhibit the same enhanced chemistry as HH 2. We thus observed several molecular lines at several positions ahead of five Herbig-Haro objects where enhanced $\mathrm{HCO}^{+}$emission was previously observed.

Methods. We mapped the five Herbig-Haro objects using the IRAM-30 m. For each position we searched for more than one molecular species, and where possible for more than one transition per species. We then estimated the averaged beam column densities for all species observed and also performed LVG analyses to constrain the physical properties of the gas.

Results. The chemically richest quiescent gas is found ahead of the HH 7-11 complex, in particular at the HH 7-11 A position. In some regions we also detected a high velocity gas component. We find that the gas densities are always higher than those typical of a molecular cloud while the derived temperatures are always quite low, ranging from 10 to $25 \mathrm{~K}$. The emission of most species seems to be enhanced with respect to that of a typical dense clump, probably due to the exposure to a high UV radiation from the HH objects. Chemical differentiation among the positions is also observed. We attempt a very simple chemical analysis to explain such differentiation.

Key words. ISM: Herbig-Haro objects - ISM: abundances - ISM: clouds - ISM: molecules - radio lines: ISM - stars: formation

\section{Introduction}

Herbig-Haro $(\mathrm{HH})$ objects are regions where protostellar jets interact hydro-dynamically with the surrounding molecular cloud, resulting in strong atomic line emission characteristic of shocks. There is now ample and increasing evidence that $\mathrm{HH}$ objects also have a less direct but very diverse chemical influence on nearby molecular clumps which have not yet been reached completely by the flow itself. In fact, the radiation generated in the $\mathrm{HH}$ objects, as well as the shocks produced by the $\mathrm{HH}$ objects alter the chemical composition of the molecular gas surrounding them in a very complex way. An example of the complex interaction of the $\mathrm{HH}$ object with its surroundings, and the most studied one, is the $\mathrm{HH} 2$ region (e.g. Girart et al. 2002; Dent et al. 2003; Lefloch et al. 2005; Girart et al. 2005). Some of the gas ahead of the HH object seems to be altered solely by the UV radiation: in the last

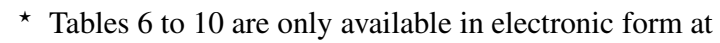
http://www. edpsciences.org decade or so quiescent, cold, molecular condensations have been found ahead of several $\mathrm{HH}$ objects. These cool condensations were found in enhanced emission of $\mathrm{HCO}^{+}$and $\mathrm{NH}_{3}$, which are tracers of high density gas (e.g. Rudolph et al. 1988; Torrelles et al. 1992; Girart et al. 1994). These emissions probably arose because the UV radiation generated in the HH shock evaporated the icy mantles on dust grains in small density enhancements in the cloud near to the head of the jet (Girart et al. 1994). Theoretical and further observational studies (Viti \& Williams 1999, hereafter VW99; Viti et al. 2003 and Girart et al. 2005) confirmed this picture and showed that, because of the quiescent nature of such clumps (clear from the linewidths of the emission as well as the derived kinetic temperature of the gas), the high abundances of $\mathrm{HCO}^{+}$and other species could not be due to shock chemistry.

In this paper, we are particularly interested in these anomalous, quiescent enhanced molecular emissions from localized regions in the vicinity of the HH objects. The VW99 models succeeded in explaining the known enhancements of $\mathrm{HCO}^{+}$ 
and $\mathrm{NH}_{3}$ relative to $\mathrm{CS}$, predicted the amount by which these species should be increased, but also indicated other species which should be enhanced (by factors of 10-200 with respect to dark molecular clouds e.g L1554), in particular $\mathrm{CH}_{3} \mathrm{OH}, \mathrm{H}_{2} \mathrm{~S}$, $\mathrm{SO}, \mathrm{SO}_{2}, \mathrm{H}_{2} \mathrm{CO}, \mathrm{C}_{3} \mathrm{H}_{4}, \mathrm{H}_{2} \mathrm{CS}$. Such predictions were confirmed by recent observations and further modelling of $\mathrm{HH} 2$ (Girart et al. 2002; Viti et al. 2003; Girart et al. 2005). It is important to note however that while single dish observations (Girart et al. 2002; Viti et al. 2003) show that the chemistry of the illuminated quiescent clumps ahead of the HH 2 object is essentially consistent with the UV field from the HH 2 front releasing the grain mantles and hence enhancing the gas chemistry, BIMA maps of such region in several molecules expected to be enhanced (Girart et al. 2005) have shown how the HH 2 object affects the chemistry and dynamic of the surrounding environment in different degrees. In summary, although Girart et al. (2005) concluded that a very complex morphological kinematical and chemical structure of the molecular gas is present ahead of the HH 2 object, they also confirmed that there is indeed an apparently quiescent molecular component that is more exposed to the UV radiation from the HH 2 front; the analysis of observational molecular lines from this region can be explained by UV radiation from the HH shock lifting the ice mantles from grains of a multi-density component clump and the consequent chemistry that arises is as complex and varied as indicated in the VW99 models.

The question we attempt to answer in this paper is the following: is the rich photochemistry characteristic of UV illumination peculiar to the $\mathrm{HH} 2$ or is it common to all $\mathrm{HH}$ objects? The simplest and most direct way to answer such question is to perform a survey of molecular species predicted to be enhanced by the VW99, ahead of several HH objects where enhanced quiescent $\mathrm{HCO}^{+}$and/or $\mathrm{NH}_{3}$ have already been detected. We have now performed such a survey with the IRAM $30-\mathrm{m}$ and we report the observational results in this paper. The main aims of this paper are: 1) determine whether other $\mathrm{HH}$ objects exhibit the same peculiar enhanced chemistry of $\mathrm{HH} 2$ as predicted by VW99 and 2) assuming the HH object to be the source of the UV radiation, look for correlations between the enhanced molecular emission and the distance of such emission to the HH object.

We report our observations in Sect. 2; in Sect. 3 we present our results and the derivation of beam averaged column densities. In Sect. 4 we analyse our data by means of radiative transfer modelling. In Sect. 5 we briefly discuss the chemical characteristics of the gas and in Sect. 6 we summarize our findings.

\section{Observations}

The observations were carried out with the IRAM $30 \mathrm{~m}$ radio telescope on Pico Veleta in November 2000. Our sample consisted of five Herbig Haro regions; for most of them we took several positions where enhanced $\mathrm{HCO}^{+}$was previously detected (see individual subsections and Table 1). For each position we searched for several molecular emissions; when possible we searched for more than one transition of the same species (see Table 2). The molecular species chosen are those which were expected from VW99 models to be enhanced
Table 1. Source list.

\begin{tabular}{c|ccc}
\hline \hline Object & $\begin{array}{c}\text { RA } \\
\text { J2000 }\end{array}$ & $\begin{array}{c}\text { Dec } \\
\text { J2000 }\end{array}$ & $\begin{array}{c}V_{\text {LSR }} \\
\mathrm{km} \mathrm{s}^{-1}\end{array}$ \\
\hline HH 7-11 A & $03: 29: 06.5$ & $31: 15: 35.5$ & +8.0 \\
HH 7-11 B & $03: 29: 06.9$ & $31: 15: 47.4$ & +8.0 \\
HH 7-11 X & $03: 29: 05.7$ & $31: 15: 30.5$ & +8.0 \\
HH 1 & $05: 36: 20.2$ & $-06: 45: 01.3$ & +9.0 \\
HH 34 A & $05: 35: 34.3$ & $-06: 29: 07.0$ & +8.5 \\
HH 34 C & $05: 35: 32.2$ & $-06: 29: 04.9$ & +8.5 \\
HH 34 D & $05: 35: 32.0$ & $-06: 29: 23.9$ & +8.5 \\
HH 34 E & $05: 35: 30.6$ & $-06: 29: 03.8$ & +8.5 \\
JMG 99 G1 & $06: 41: 04.5$ & $09: 56: 02.0$ & +4.6 \\
JMG 99 G2 & $06: 41: 07.1$ & $09: 56: 07.0$ & +4.0 \\
\hline
\end{tabular}

(with respect to dark molecular clouds) by reasonably large factors, along with tracers of the underlying column density in the cloud (CS and CO isotopes) and tracers of the ionization (the ratio $\mathrm{DCO}^{+} / \mathrm{HCO}^{+}$; Caselli et al. 1998). Due to time constraints, we could not attempt to detect all the species predicted by the VW99 at every position. Our criteria varied from object to object but in general if $\mathrm{HCO}^{+}$emission was weak we did not attempt a detection in other species. We also prioritized species easier to observe, so for example, although both $\mathrm{SO}$ and $\mathrm{SO}_{2}$ are predicted by the models to be enhanced we concentrated on SO as its lines are usually stronger and also it has a higher predicted abundance. All the transitions were observed in the frequency bands of 100, 150, 230 and $270 \mathrm{GHz}$ (see Table 2 for line frequencies). Integration times were typically $2-12 \mathrm{~min}$ (position switched), with longer attempts at detecting weak lines (e.g. NS: 30 mins). The spectral resolution was $40 \mathrm{kHz}$ $(100 / 150 \mathrm{GHz}$ band) or $80 \mathrm{kHz}(230 / 270 \mathrm{GHz}$ band) resulting in a velocity resolution typically of $0.05 \mathrm{~km} \mathrm{~s}^{-1}$ and at most $0.13 \mathrm{~km} \mathrm{~s}^{-1}$. The bandwidth was at least $20 \mathrm{MHz}(100 / 150 \mathrm{GHz}$ bands) or $40 \mathrm{MHz}$ (230/270 GHz bands). Conditions were generally poor and variable for winter nights with 1-6 mm PWV (precipitable water vapour). Resulting system temperatures were $T_{\text {sys }} \sim 100-120,200-460,200-300$ and 400-700 K for the $100,150,230$ and $270 \mathrm{GHz}$ bands respectively, depending on frequency and weather. Pointing was good to within 4 arcsec. Spectra were corrected for the beam efficiencies $B_{\text {eff }}=$ $0.75,0.69,0.51$, and 0.42 in the $100,150,230$, and $270 \mathrm{GHz}$ bands respectively. The $30 \mathrm{~m}$ beam $F W H M$ ranges as $v^{-1}$ from $28^{\prime \prime}$ at $86 \mathrm{GHz}$ down to $11^{\prime \prime}$ at $230 \mathrm{GHz}$. See on-line Tables 6 to 10 for details of the molecular transitions observed.

The choice of our sample of objects was made by selecting all the $\mathrm{HH}$ objects with known $\mathrm{HCO}^{+}$emission enhancements. For some objects, we detected two velocity components in several species: a high velocity one, probably coming from the closest part to the $\mathrm{HH}$ object where the gas has already been dynamically affected; and an ambient one. In this paper we will mainly concentrate on the latter. 
Table 2. Frequency setups of the IRAM $30 \mathrm{~m}$ observations and list of positions observed.

\begin{tabular}{|c|c|c|c|c|c|c|c|c|c|c|c|c|}
\hline \multirow{2}{*}{$\begin{array}{l}\text { Molecular } \\
\text { Transitions }\end{array}$} & \multirow{2}{*}{$\begin{array}{l}\text { Frequency } \\
(\mathrm{GHz})\end{array}$} & \multirow{2}{*}{$\begin{array}{l}\text { Beam } \\
\left({ }^{\prime \prime}\right)\end{array}$} & \multicolumn{3}{|c|}{ HH 7-11 } & \multicolumn{4}{|c|}{ HH 34} & \multicolumn{2}{|c|}{$\mathrm{N} 2264 \mathrm{G}^{a}$} & \multirow[b]{2}{*}{ HH 1} \\
\hline & & & $\mathrm{A}$ & $X$ & B & $\mathrm{A}$ & $\mathrm{E}$ & $\mathrm{C}$ & $\mathrm{D}$ & 1 & 2 & \\
\hline${ }^{13} \mathrm{CO} 2-1$ & 220.3980 & 11.0 & $\mathrm{X}$ & $\mathrm{X}$ & $X$ & $\mathrm{X}$ & $\mathrm{X}$ & $\mathrm{X}$ & $X$ & $X$ & $X$ & $X$ \\
\hline $\mathrm{C}^{18} \mathrm{O} 2-1$ & 219.5604 & 11.0 & $X$ & $X$ & & $X$ & $\mathrm{X}$ & & & $\mathrm{X}$ & $X$ & $X$ \\
\hline $\mathrm{HCO}^{+} 1-0$ & 89.1885 & 28.0 & $X$ & $\mathrm{X}$ & $X$ & $\mathrm{X}$ & $\mathrm{X}$ & $\mathrm{X}$ & $X$ & $\mathrm{X}$ & $\mathrm{X}$ & $\mathrm{X}$ \\
\hline $\mathrm{HCO}^{+} 3-2$ & 267.5576 & 9.0 & $\mathrm{X}$ & $\mathrm{X}$ & $\mathrm{X}$ & $\mathrm{X}$ & $\mathrm{X}$ & $\mathrm{X}$ & $\mathrm{X}$ & $X$ & $\mathrm{X}$ & $\mathrm{X}$ \\
\hline $\mathrm{H}^{13} \mathrm{CO}^{+} 1-0$ & 86.7543 & 29.0 & $\mathrm{X}$ & $X$ & $\mathrm{X}$ & $\mathrm{X}$ & $\mathrm{X}$ & $X$ & $\mathrm{X}$ & $\mathrm{X}$ & $X$ & $\mathrm{X}$ \\
\hline $\mathrm{H}^{13} \mathrm{CO}^{+} 3-2$ & 260.2555 & 9.5 & $\mathrm{X}$ & $X$ & $\mathrm{X}$ & $\mathrm{X}$ & $\mathrm{X}$ & & & $X$ & $\mathrm{X}$ & $\mathrm{X}$ \\
\hline $\mathrm{DCO}^{+} 2-1$ & 144.0773 & 17.0 & $\mathrm{X}$ & $\mathrm{X}$ & & $\mathrm{X}$ & $\mathrm{X}$ & & & $\mathrm{X}$ & $\mathrm{X}$ & $X$ \\
\hline $\mathrm{DCO}^{+} 3-2$ & 216.1126 & 12.0 & $\mathrm{X}$ & $\mathrm{X}$ & & $\mathrm{X}$ & $X$ & & & $X$ & $\mathrm{X}$ & $X$ \\
\hline HCN 3-2 & 265.8864 & 9.0 & $X$ & $X$ & & $\mathrm{X}$ & $\mathrm{X}$ & & & $X$ & $X$ & $X$ \\
\hline $\mathrm{H}_{2} \mathrm{CO} 2_{0,2}-1_{0,1}$ & 145.6029 & 17.0 & $\mathrm{X}$ & $\mathrm{X}$ & & $\mathrm{X}$ & $\mathrm{X}$ & & & & & $X$ \\
\hline $\mathrm{H}_{2} \mathrm{CO} 3_{1,3}-2_{1,2}$ & 211.2115 & 12.0 & $X$ & & & & $\mathrm{X}$ & & & & & \\
\hline $\mathrm{CH}_{3} \mathrm{OH} 2_{k}-1_{k}^{b}$ & 96.7414 & 26.0 & $X$ & $\mathrm{X}$ & & $\mathrm{X}$ & $\mathrm{X}$ & & & $X$ & $\mathrm{X}$ & \\
\hline $\mathrm{CH}_{3} \mathrm{OH} 3_{k}-2_{k}{ }^{c}$ & 145.1032 & 17.0 & $\mathrm{X}$ & $\mathrm{X}$ & & $\mathrm{X}$ & $\mathrm{X}$ & & & $X$ & $X$ & \\
\hline $\mathrm{CH}_{3} \mathrm{OH} 5_{k}-4_{k}^{d}$ & 241.7914 & 10.0 & $X$ & $\mathrm{X}$ & $X$ & & & & & & & \\
\hline $\mathrm{C}_{3} \mathrm{H}_{4} 5_{0}-4_{0}$ & 85.4573 & 29.0 & $\mathrm{X}$ & & & & $\mathrm{X}$ & & & & & \\
\hline $\mathrm{C}_{3} \mathrm{H}_{2} 2_{1,2}-1_{0,1}$ & 85.3389 & 29.0 & $\mathrm{X}$ & & & & $\mathrm{X}$ & & & & & \\
\hline $\mathrm{C}_{3} \mathrm{H}_{2} 3_{1,2}-2_{2,1}$ & 145.0896 & 29.0 & $\mathrm{X}$ & $X$ & & & & & & & & \\
\hline $\mathrm{C}_{3} \mathrm{H}_{2} 4_{1,4}-3_{0,3}$ & 150.8519 & 17.0 & $X$ & & & & & & & & & \\
\hline CS 3-2 & 146.9690 & 17.0 & $\mathrm{X}$ & $X$ & $\mathrm{X}$ & $\mathrm{X}$ & $\mathrm{X}$ & $\mathrm{X}$ & $\mathrm{X}$ & $\mathrm{X}$ & $\mathrm{X}$ & $\mathrm{X}$ \\
\hline CS 5-4 & 244.9356 & 10.0 & $\mathrm{X}$ & & & & $\mathrm{X}$ & & & & & \\
\hline $\mathrm{SO} 3_{2}-2_{1}$ & 99.2999 & 20.0 & $X$ & $\mathrm{X}$ & & $\mathrm{X}$ & $\mathrm{X}$ & & & $X$ & $\mathrm{X}$ & $X$ \\
\hline SO $6_{5}-5_{4}$ & 219.9494 & 11.5 & $X$ & $X$ & & $X$ & $\mathrm{X}$ & & & $X$ & $X$ & $X$ \\
\hline $\mathrm{SO} 7_{6}-6_{5}$ & 261.8437 & 9.5 & $\mathrm{X}$ & $\mathrm{X}$ & & $\mathrm{X}$ & $\mathrm{X}$ & & & $X$ & $\mathrm{X}$ & $\mathrm{X}$ \\
\hline $\mathrm{H}_{2} \mathrm{~S} 1_{1,0}-0_{0,1}$ & 168.7628 & 14.5 & $X$ & $X$ & $X$ & & & & & $X$ & $X$ & $X$ \\
\hline $\mathrm{H}_{2} \mathrm{CS} 4_{0,4}-3_{0,3}$ & 137.3711 & 18.0 & $\mathrm{X}$ & & & & $\mathrm{X}$ & & & & & \\
\hline $\mathrm{HCS}^{+} 5-4$ & 213.3606 & 12.0 & $X$ & & & & & & & & & \\
\hline NS $7 / 2-5 / 2$ & 161.2972 & 15.0 & $\mathrm{X}$ & & & & $\mathrm{X}$ & & & & & \\
\hline OCS 7-6 & 85.1391 & 29.0 & $X$ & & & & & & & & & \\
\hline
\end{tabular}

${ }^{a}$ Abbreviation of NGC 2264G JMG1 and JMG2.

${ }^{b} 2_{0}-1_{0} \mathrm{~A}^{+}$transition.

${ }^{c} 3_{0}-2_{0} \mathrm{~A}^{+}$transition.

${ }^{d} 5_{0}-4_{0} \mathrm{~A}^{+}$transition.

\subsection{Brief description of the sources}

\subsection{1. $\mathrm{HH} 7-11$}

The HH 7-11 system $(d \sim 220$ pc: Cernis 1990) consists of fairly low excitation $\mathrm{HH}$ objects, with $\mathrm{HH} 7$ being at the far end of the outflow (Solf et al. 1987). However, the region surrounding HH 7-11 is very rich in young stellar objects (e.g. Rodríguez et al. 1999), hence the total radiation impinging on the surroundings is unknown. Enhanced $\mathrm{HCO}^{+} 1-0$ emission has been previously observed just ahead of $\mathrm{HH}$ 8-10 objects in the form of small clumps with 7" sizes (1500 AU, Rudolph et al. 1988). CS and $\mathrm{NH}_{3}$ (Rudolph et al. 2001) are also reported towards these clumps. Submillimeter observations in the region reveal a dust source, HH 7-11 MMS 4, that engulfs the $\mathrm{HCO}^{+}$clumps (Chini et al. 2001). From the dust observations Chini et al. (2001) derive that the total mass of the quiescent gas around $\mathrm{HH} 8-10$ is $4.0 M_{\odot}$. From the study of the $\mathrm{HCO}^{+} 3-2$ emission, Dent et al. (1993) suggest that the dense molecular gas and dust around $\mathrm{HH} 8-11$ is the densest, slowest component of the outflow. This is somewhat similar to the picture of the molecular gas ahead of HH 2 (Girart et al. 2005).

We selected three positions, two of them, HH 7-11 A and $\mathrm{B}$, toward the $\mathrm{HCO}^{+} 1-0$ clumps (Rudolph et al. 1988) and one, $\mathrm{HH}$ 7-11 X, toward a $\mathrm{HCO}^{+} 3-2$ emission peak southwest of HH 8 (Dent et al. 1993). Note that the $3 \mathrm{~mm}$ beams of our 3 positions (A, X and B) intersect (see Fig. 1).

\subsection{2. $\mathrm{HH} 1$}

The HH 1-2 system $(d \sim 450 \mathrm{pc})$ is one of the most intensely studied $\mathrm{HH}$ object system to date. The proper motions of HH 1 and HH 2 show that they are moving in opposite 


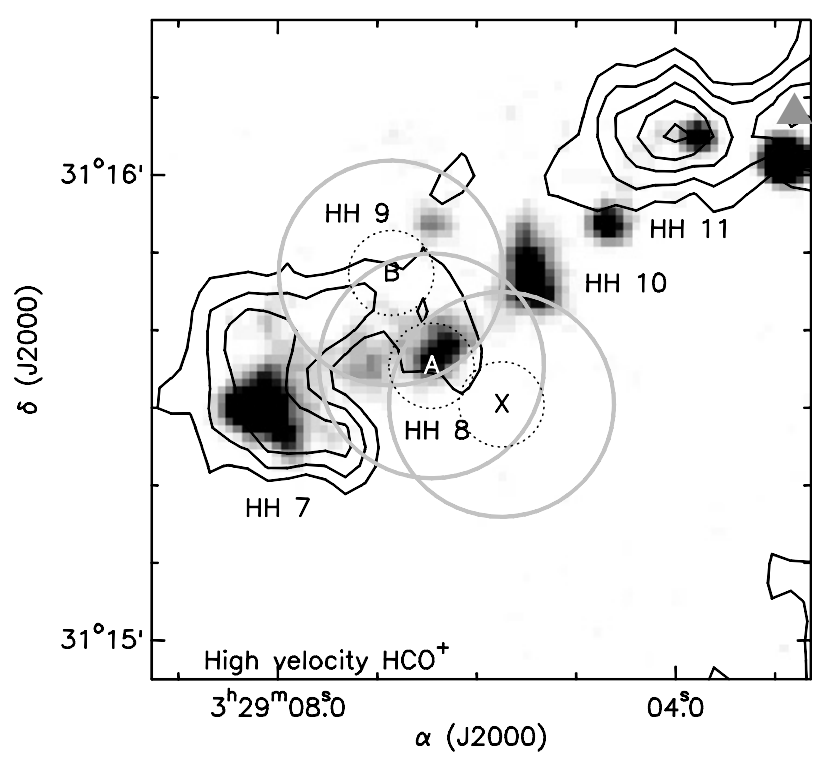

Fig. 1. Sketch of the HH 7-11 region. The gray scale shows the optical $R$ band image of the $\mathrm{HH} 7-11$ objects, while the contour plots shows the BIMA $\mathrm{HCO}^{+} 1-0$ blueshifted high velocity emission (from Mehringer 1996). The optical image was taken from the public cata$\log$ Aladin. The circles are the IRAM $1 \mathrm{~mm}$ (dotted) and $3 \mathrm{~mm}$ (solid) beams at the $\mathrm{A}, \mathrm{B}$ and $\mathrm{X}$ positions.

directions (Herbig \& Jones 1981). Their powering source is probably the embedded luminous young star known as HH 1-2 VLA 1 (Pravdo et al. 1985). Infrared and optical images show very complex structures, quite randomly positioned in the case of $\mathrm{HH} 2$ and more like a set of bow shock features in $\mathrm{HH} 1$. Clumpy molecular emission ahead of HH 2 has been the subject of an extensive recent study (Girart et al. 2005). Enhanced $\mathrm{HCO}^{+}$and $\mathrm{NH}_{3}$ have also being previously observed ahead of HH 1 (Davis et al. 1990; Torrelles et al. 1993). No dust is detected towards the $\mathrm{NH}_{3}$ clumps (Chini et al. 2001) up to $0.22 \mathrm{Jy}$ beam $^{-1}(3-\sigma)$ at $850 \mu \mathrm{m}$. Note that the dust peak emission ahead of HH 2 is $0.20 \pm 0.04 \mathrm{Jy}$ (Dent et al. 2003). Our IRAM 30 m observations pointed toward the $\mathrm{NH}_{3}(1,1)$ ammonia peak ahead of HH 1 (see Fig. 2). We did a search for the most abundant species towards this region.

\subsection{3. $\mathrm{HH} 34$}

The HH 34 is a high excitation large bow shock (Reipurth et al. 2002), which is part of a parsec scale HH system located in the molecular cloud L1641 in Orion $(d \sim 480 \mathrm{pc})$. Its source may be a faint star at $46000 \mathrm{AU}$ away. Enhanced, and quiescent, $\mathrm{HCO}^{+} 1-0$ emission was detected from five clumps with sizes of about $15^{\prime \prime}$ or $7000 \mathrm{AU}$ (Rudolph et al. 1992). The clumps are located south of HH 34 at a distance to the HH object of 0.04-0.09 pc, corresponding to 8000-18000 AU. We have searched for the predicted set of molecules at four positions which coincide with the peak $\mathrm{HCO}^{+}$emission of four of the detected clumps, A, E, C and D, by Rudolph et al. (1992; see Fig. 3), although for these two last positions only ${ }^{13} \mathrm{CO}, \mathrm{HCO}^{+}$and $\mathrm{CS}$ were observed.

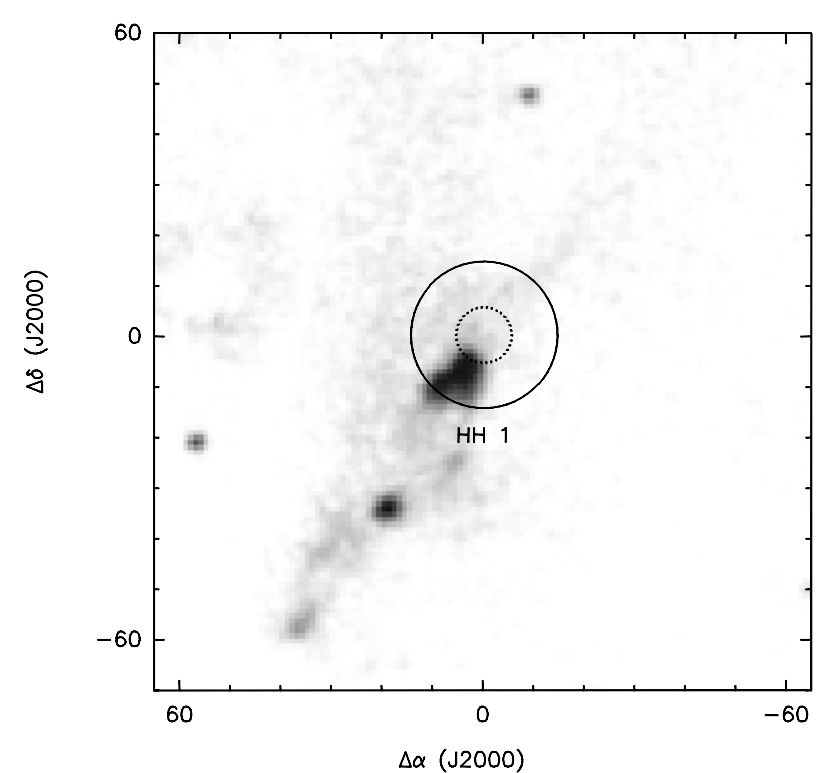

Fig. 2. Sketch of the HH 1 region. The gray scale shows the optical $R$ band image of the HH 1 object, taken from the public catalog Aladin. The circles are the IRAM $1 \mathrm{~mm}$ (dotted) and $3 \mathrm{~mm}$ (solid) beams.

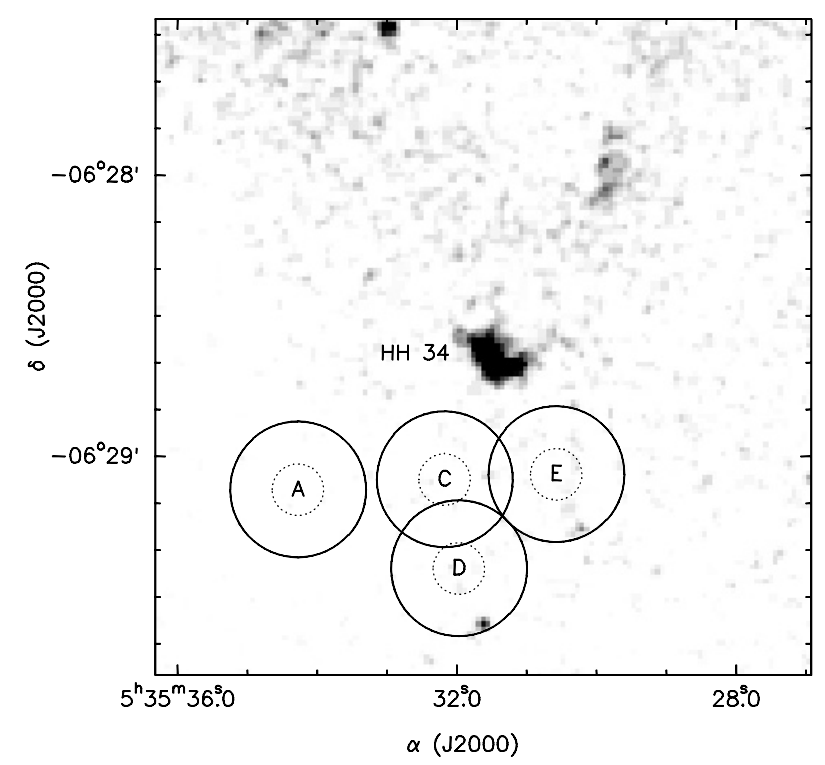

Fig. 3. Sketch of the HH 34 region. The grey scale shows the optical $R$ band image of the HH 34 object, taken from the public catalogue Aladin. The circles are the IRAM $1 \mathrm{~mm}$ (dotted) and $3 \mathrm{~mm}$ (solid) beams at the HH $34 \mathrm{~A}, \mathrm{C}, \mathrm{D}$ and $\mathrm{E}$ positions.

\subsubsection{NGC $2264 G$}

NGC 2264G is associated with a spectacular CO outflow driven by a Class 0 protostar (Lada et al. 1996; Gómez et al. 1994; Ward-Thompson et al. 1995). Girart et al. (2000) found two small clumps (sizes of $\$ 14^{\prime \prime}$ ) of apparently quiescent $\mathrm{HCO}^{+}$4-3 emission, JMG 99 G1 and JMG 99 G2, west of the protostar and just ahead from shock-excited near-IR emission. Recent observations show that these clumps are also associated with strong high velocity $\mathrm{SiO}$ and $\mathrm{CH}_{3} \mathrm{OH}$ emission (Garay et al. 2002). We observed these two clumps. 


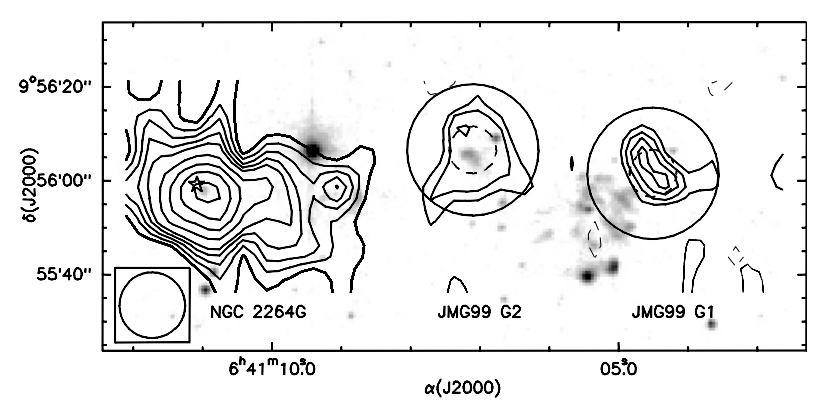

Fig. 4. JCMT contour maps of the integrated $\mathrm{HCO}^{+} 4-3$ emission (from Girart et al. 2000) overlapped with the grey scale image of the near-IR $\mathrm{H}_{2}$ emission. The circles are the IRAM $1 \mathrm{~mm}$ (dashed) and $3 \mathrm{~mm}$ (solid) beams at the JMG99 G2 and G1 positions.

\section{Results}

Figure 5 shows examples of the spectra of the molecular transitions for which emission was detected. HH 7-11 is the region where molecular lines are brighter so position $\mathrm{HH} 7-$ 11 A was selected to carry out the most complete survey. Apart from $\mathrm{HCS}^{+}$, all the molecular species predicted by the VW99 to be enhanced were detected toward this position. All the positions observed but JMG 99 G1 shows that the emission is dominated by relatively narrow lines (mostly between 0.4 and $1.5 \mathrm{~km} \mathrm{~s}^{-1}$ ), typical of quiescent emission, although line width can vary from region to region or between different species (see on-line Tables 6-10). HH 1 shows the largest differences between line widths for different species, ranging from $\sim 0.5 \mathrm{~km} \mathrm{~s}^{-1}$ for $\mathrm{DCO}^{+}$to $2.4 \mathrm{~km} \mathrm{~s}^{-1}$ for $\mathrm{CH}_{3} \mathrm{OH}$. These large variations are also observed in the $\mathrm{HH} 2$ molecular clumps (Girart et al. 2002). For HH 7-11 positions and JMG $99 \mathrm{G} 2$ most line widths are in the 0.8 to $1.4 \mathrm{~km} \mathrm{~s}^{-1}$ range. Interestingly, for these positions the HCN 3-2 line emission is significantly broader than the averaged line width (roughly by a factor 2). ${ }^{13} \mathrm{CO}$ lines are in most positions either clearly nonGaussian or have several components, which can be explained from contribution from lower density components along the line of sight. In these cases, in order to estimate the integrated line intensity, we selected the velocity range where the higher density tracer showed up. Strong absorption is observed at the center of the $\mathrm{HCO}^{+} 1-0$ line in the $\mathrm{HH}$ 7-11 and specially in the NGC $2264 \mathrm{G}$ region, which is due to the presence of a low density molecular gas in the foreground (Girart et al. 2000).

Some of the observed positions show high velocity wings in the $\mathrm{HCO}^{+} 1-0$ line (the three positions in $\mathrm{HH} \mathrm{7-11,} \mathrm{HH}$, HH 34 E and JMG 99 G2). This is more clearly seen in HH 711 A (see Fig. 5), where other strong lines also show wings: ${ }^{13} \mathrm{CO}, \mathrm{H}_{2} \mathrm{CO}, \mathrm{CS}, \mathrm{CH}_{3} \mathrm{OH}$.

The properties of the molecular emission toward JMG 99 G1 is quite different from the other regions. Some of the molecular transitions have emission at the systemic velocity of the cloud (e.g. $\mathrm{C}^{18} \mathrm{O} 2-1, \mathrm{HCO}^{+} 4-3, \mathrm{CS} 3-2$ ) but the strongest emission arises from a broad, $\Delta v \simeq 5 \mathrm{~km} \mathrm{~s}^{-1}$, blueshifted component. This component is also observed in $\mathrm{SiO}$ (Garay et al. 2002). All these features indicate that most of the molecular emission in JMG 99 G1 comes from shocked outflow material (see Sect. 5.3).

\section{Analysis}

The excitation temperature and the optical depth for the $\mathrm{HCO}^{+}$ can be derived from the line ratio of the different $\mathrm{HCO}^{+}$and $\mathrm{H}^{13} \mathrm{CO}^{+}$observed transitions. From this analysis we found that $T_{\text {ex }} \simeq 6-8 \mathrm{~K}$ and $\tau\left(\mathrm{HCO}^{+} 3-2\right) \simeq 3-11$. Therefore $\mathrm{HCO}^{+}$beam averaged column density is derived adopting an excitation temperature of $7 \mathrm{~K}$ and correcting for the optical depth. For species which also have a high dipole moment (e.g. $\mathrm{HCN}, \mathrm{H}_{2} \mathrm{CO}$ ) the column densities are estimated assuming LTE and adopting $T_{\mathrm{ex}}=7 \mathrm{~K}$ but also assuming that the emission is optically thin. For the other species we adopt $T_{\mathrm{ex}}=10 \mathrm{~K}$. Column densities of $\mathrm{CO}$ and isotopes are corrected for optical depth. For a non detection we report the $3-\sigma$ upper limit. Table 3 shows the derived beam averaged column densities for the quiescent emission (i.e. excluding the high velocity component) for all the positions but JMG 99 G1. For reference, we also include the column densities in the $\mathrm{SO}_{2}$ clump ahead of $\mathrm{HH} 2$ taken from Girart et al. (2005). Note that Girart et al. obtained column densities for several positions along the slice in the $\mathrm{SO}_{2}$ clump (see their Table A.2); we chose to present only one of them (position I3, $T_{\mathrm{ex}}=13 \pm 2 \mathrm{~K}$ ). Most of the lines observed toward JMG 99 G1 show emission that is more characteristic of strongly perturbed gas. In addition we used the LVG and RADEX codes to carry out a more detailed analysis for the molecules with several transitions observed (see Table 4).

For the high velocity emission toward HH 7-11 and JMG 1 we also estimated the column densities assuming LTE and adopting $T_{\mathrm{ex}}=30 \mathrm{~K}$. In these cases, we computed the column density in different velocity intervals along the velocity range where the high velocity is detected (see Sect. 5.3).

\subsection{LVG and RADEX analysis}

In this section we estimate, to a first approximation, some of the physical and chemical characteristics of most clumps, such as the density and the temperature by means of large velocity gradient (LVG) and RADEX modelling.

We observed multitransition observations of $\mathrm{HCO}^{+}$and $\mathrm{H}^{13} \mathrm{CO}^{+}$for $\mathrm{HH}$ 7-11 A, HH 7-11 B and $\mathrm{HH}$ 7-11 X, HH 1, $\mathrm{HH} 34 \mathrm{~A}$ and $\mathrm{HH} 34 \mathrm{E}$. We therefore carried out radiative transfer calculations using the LVG model approximation. The main goal of the modelling is to constrain the range of densities and temperatures of the clumps, as well as the $\mathrm{HCO}^{+}$true column densities. LVG modelling is performed varying three parameters: the column density of $\mathrm{HCO}^{+}$, the temperature, and the volume density of the gas; one parameter is provided as an input, and the aim is to obtain a set of LVG solutions in the other two parameters plane. For $\mathrm{HCO}^{+}$, we ran the $\mathrm{LVG}$ code with the $\mathrm{HCO}^{+}$column density as input parameter and we stopped when a set of reasonable solutions was obtained. To constrain the set of solutions we used all the observed line intensity as well as the observed line temperature ratios $\left(\left[\mathrm{H}^{12} \mathrm{CO}^{+} 3-\right.\right.$ $2] /\left[\mathrm{H}^{13} \mathrm{CO}^{+} 3-2\right]$ and $\left.\left[\mathrm{H}^{13} \mathrm{CO}^{+} 2-1\right] /\left[\mathrm{H}^{13} \mathrm{CO}^{+} 3-2\right]\right)$. To account for the difference in beam sizes between the different transitions, we multiplied our intensities and temperature ratios by the appropriate filling factor (assuming that the size of the emission is the same for all the transitions and that the 

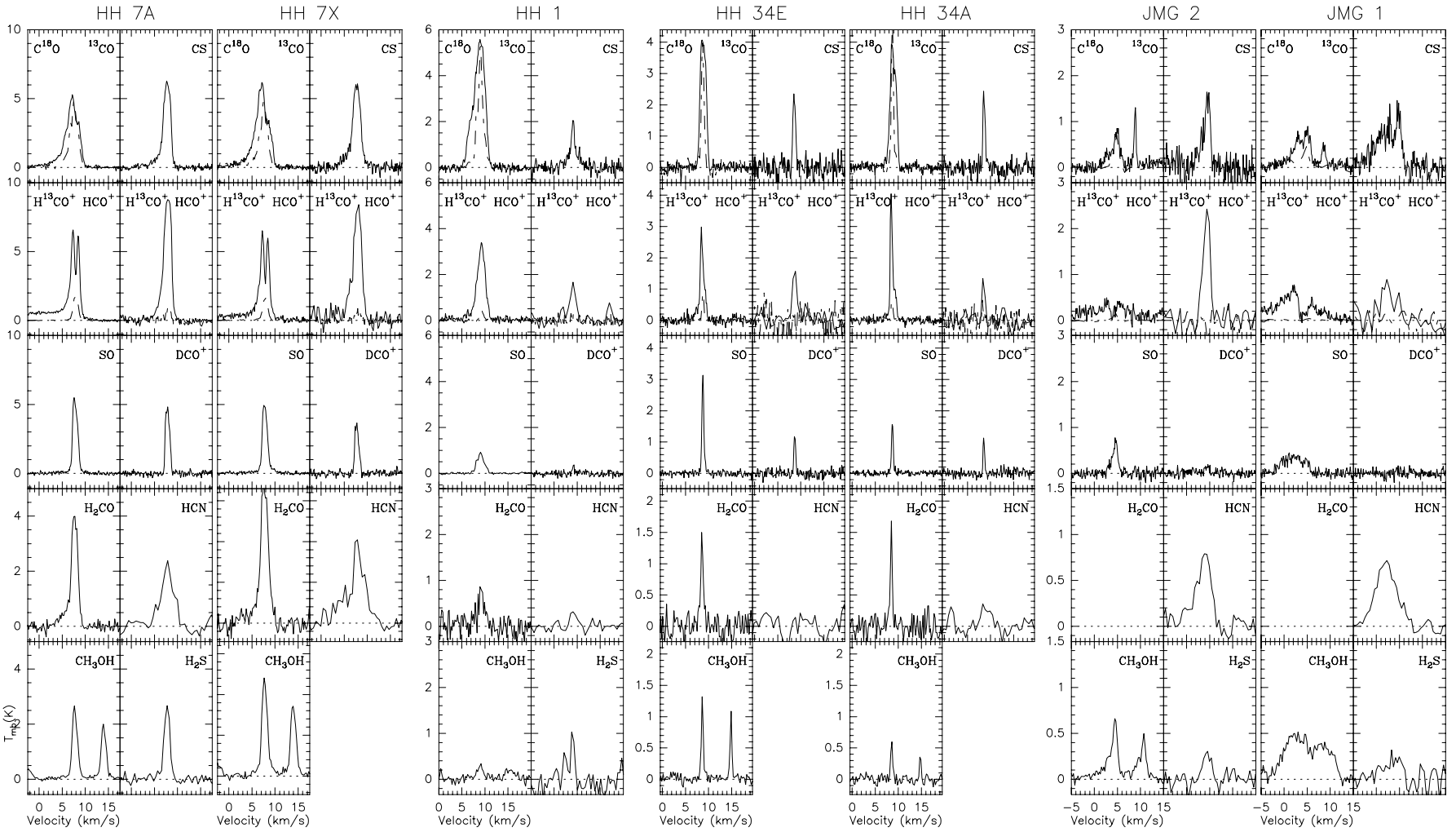

Fig. 5. Spectra of selected transitions of the different species observed toward (from left to right) HH 7-11 A, HH 7-11X, HH 1, HH 34E, HH 34A, JMG 2 and JMG $1 .{ }^{13} \mathrm{CO} 2-1$ spectra is scaled down by a factor of 3 to fit in the boxes. The transition lines showed are (from left to right and from top to bottom ): ${ }^{13} \mathrm{CO} 2-1, \mathrm{CS} 3-2, \mathrm{HCO}^{+} 1-0, \mathrm{HCO}^{+} 3-2, \mathrm{SO}_{3}-2_{1}, \mathrm{DCO}^{+} 2-1, \mathrm{H}_{2} \mathrm{CO}_{2,2}-1_{0,1}, \mathrm{HCN}_{3}-2, \mathrm{CH}_{3} \mathrm{OH} 2_{K}-1_{K}$, and $\mathrm{H}_{2} \mathrm{~S}_{1,0}-0_{0,1}$. The dashed lines in the $\mathrm{CO}$ and $\mathrm{HCO}^{+}$panels are for the $\mathrm{C}^{18} \mathrm{O}$ and $\mathrm{HC}^{13} \mathrm{CO}^{+}$isotopes at the same transition, respectively.

Table 3. Averaged beam column densities of the low velocity component in the clumps ahead of HH objects observed with IRAM $30 \mathrm{~m}$ (Cols. 2-10); the last column shows, for reference, the column densities (when available) at position $\mathrm{I} 3$ ahead of HH2 taken from Girart et al. (2005, Table A.2). To fit the table, we abbreviated HH 7-11 to 7-11, the HH 34 clumps to HH34, and NGC 2264 G JM G2 to JMG 99 G2. $a(b)$ stands for $a \times 10^{b}$.

\begin{tabular}{ccccccccccc}
\hline \hline MOL. & $7-11 \mathrm{~A}$ & $7-11 \mathrm{X}$ & $7-11 \mathrm{~B}$ & $\mathrm{HH} 34 \mathrm{~A}$ & $\mathrm{HH} 34 \mathrm{E}$ & $\mathrm{HH} 34 \mathrm{C}$ & HH34 D & HH 1 & JMG 99 G2 & HH 2 \\
\hline $\mathrm{CO}^{a}$ & $2.6(18)$ & $2.3(18)$ & $2.1(18)$ & $1.9(18)$ & $1.4(18)$ & $1.3(18)$ & $1.2(18)$ & $3.5(18)$ & $3.7(17)$ & $3.3(17)$ \\
${ }^{13} \mathrm{CO}$ & $4.6(16)$ & $4.0(16)$ & $3.7(16)$ & $3.0(16)$ & $2.2(16)$ & $2.0(16)$ & $1.9(16)$ & $5.5(16)$ & $6.5(15)$ & - \\
$\mathrm{CH}_{3} \mathrm{OH}$ & $3.5(14)$ & $4.1(14)$ & - & $2.9(13)$ & $4.9(13)$ & - & - & $5.6(13)$ & $9.8(13)$ & $6.2(13)$ \\
$\mathrm{HCO}^{+b}$ & $2.0(14)$ & $1.6(14)$ & $2.2(14)$ & $1.2(13)$ & $3.3(13)$ & $1.7(13)$ & $9.7(12)$ & $2.1(13)$ & $5.0(12)$ & $4.0(13)$ \\
$\mathrm{H}^{13} \mathrm{CO}^{+}$ & $2.3(12)$ & $1.8(12)$ & $2.5(12)$ & $1.5(12)$ & $4.2(12)$ & - & - & $2.6(12)$ & $6.0(11)$ & - \\
$\mathrm{SO}$ & $8.5(13)$ & $8.6(13)$ & - & $8.2(12)$ & $1.7(13)$ & - & - & $1.7(13)$ & $1.6(13)$ & $1.7(13)$ \\
$\mathrm{H}_{2} \mathrm{CO}^{c}$ & $9.3(13)$ & $9.6(13)$ & - & $1.0(13)$ & $1.1(13)$ & - & - & $1.6(13)$ & - & $9.0(13)$ \\
$\mathrm{CS}$ & $6.0(13)$ & $6.2(13)$ & $5.9(13)$ & $6.8(12)$ & $8.2(12)$ & $6.2(12)$ & $5.6(12)$ & $1.0(13)$ & $1.1(13)$ & $2.0(12)$ \\
$\mathrm{H}_{2} \mathrm{~S}$ & $5.0(13)$ & $4.7(13)$ & $4.5(13)$ & - & - & - & - & $1.2(13)$ & $6.0(12)$ & - \\
$\mathrm{C}_{3} \mathrm{H}_{4}$ & $5.7(13)$ & - & - & - & $<2(13)$ & - & - & - & - & - \\
$\mathrm{HCN}^{2}$ & $2.3(13)$ & $1.6(13)$ & - & $<6(11)$ & $<6(11)$ & - & - & $1.8(12)$ & $7.8(12)$ & $2.8(12)$ \\
$\mathrm{OCS}^{2.1(13)}$ & - & - & - & - & - & - & - & - & - \\
$\mathrm{H}_{2} \mathrm{CS}$ & $1.1(13)$ & - & - & - & $<2(12)$ & - & - & - & - & - \\
$\mathrm{C}_{3} \mathrm{H}_{2}$ & $8.9(12)$ & $8.4(12)$ & - & - & $8.5(11)$ & - & - & - & - & - \\
$\mathrm{NS}^{7.8(12)}$ & - & - & - & $<5(12)$ & - & - & - & - & - \\
$\mathrm{DCO}^{+}$ & $5.0(12)$ & $3.6(12)$ & - & $3.8(11)$ & $2.9(11)$ & - & - & $1.9(11)$ & $2.2(11)$ & $7.8(11)$ \\
$\mathrm{HCS}^{+}$ & $<2(12)$ & - & - & - & - & - & - & - & - & - \\
\hline
\end{tabular}

${ }^{a}$ Optical depth of HH 7-11 B adopted from HH 7-11 X, and of HH 34 C and HH 34 D adopted from HH 34 E.

${ }^{b}$ Optical depth of HH $34 \mathrm{C}$ and HH 34 D adopted from HH 34 E.

${ }^{c}$ Adopted an ortho/para ratio of 1.5 . 
Table 4. Best fits with LVG and RADEX for column densities, gas densities and temperatures. $a(b)$ stands for $a \times 10^{b}$.

\begin{tabular}{ccccc}
\hline \hline HCO & \multicolumn{5}{c}{} \\
\hline Object & Size & $N\left(\mathrm{HCO}^{+}\right)\left(\mathrm{cm}^{-2}\right)$ & $n\left(\mathrm{H}_{2}\right)\left(\mathrm{cm}^{-3}\right)$ & $T_{k}(\mathrm{~K})$ \\
\hline HH 7-11 A & $20^{\prime \prime}$ & $5(14)-1(15)$ & $8(4)-3(5)$ & $15-24$ \\
HH 7-11 B & $20^{\prime \prime}$ & $5(14)-1(15)$ & $9(4)-4(5)$ & $15-24$ \\
HH 7-11 X & $20^{\prime \prime}$ & $5(14)-1(15)$ & $3(5)-2(6)$ & $7-11$ \\
HH 1 & $10^{\prime \prime}$ & $5(14)-1(15)$ & $4(5)-1(6)$ & $8-11$ \\
HH 34 A & $10-15^{\prime \prime}$ & $5(14)-1(15)$ & $3-4(5)$ & $7-11$ \\
HH 34 E & $10-12^{\prime \prime}$ & $5(14)-1(15)$ & $1.5-9(5)$ & $8-12$ \\
\hline \multicolumn{5}{c}{$\mathrm{CS}$} \\
\hline Object & Size & $N(\mathrm{CS})\left(\mathrm{cm}^{-2}\right)$ & $n\left(\mathrm{H}_{2}\right)\left(\mathrm{cm}^{-3}\right)$ & $T_{k}(\mathrm{~K})$ \\
\hline HH 7-11 A & $30^{\prime \prime}$ & $5(14)-1(15)$ & $1(5)-3(5)$ & 15 \\
HH 34 E & $30^{\prime \prime}$ & $3(13)-1(14)$ & $3(5)-9(5)$ & $10-12$ \\
\hline \multicolumn{5}{c}{$\mathrm{SO}$} \\
\hline Object & Size & $N(\mathrm{SO})\left(\mathrm{cm}^{-2}\right)$ & $n\left(\mathrm{H}_{2}\right)\left(\mathrm{cm}^{-3}\right)$ & $T_{k}(\mathrm{~K})$ \\
\hline HH 7-11 A & $20^{\prime \prime}$ & $3(14)-1(15)$ & $3(4)-4(5)$ & $>10$ \\
HH 7-11 X & $20^{\prime \prime}$ & $3(14)-1(15)$ & $2(4)-2(5)$ & $>10$ \\
\hline Object & Size & $N\left(\mathrm{C}_{3} \mathrm{H}_{2}\right)\left(\mathrm{cm}^{-2}\right)$ & $n\left(\mathrm{H}_{2}\right)\left(\mathrm{cm}^{-3}\right)$ & $T_{k}(\mathrm{~K})$ \\
\hline HH 7-11 A & $20^{\prime \prime}$ & $3(13)-1(14)$ & $4(4)-2(5)$ & $>10$ \\
\hline \multicolumn{5}{c}{}
\end{tabular}

emission has a Gaussian shape). We initially used the $\mathrm{HCO}^{+}$ size derived from previous observations (see following subsections), although we also explored different values around the reported values to check if the $\mathrm{LVG}$ fits improved. The solutions were searched in the $T_{k}-n\left(\mathrm{H}_{2}\right)$ plane for different $\mathrm{HCO}^{+}$column densities ranging from $1 \times 10^{13}$ to $1 \times 10^{15} \mathrm{~cm}^{-2}$ (the $\mathrm{HCO}^{+}$ beam averaged column densities for the clumps where we perform the LVG analysis are in the $2 \times 10^{13}-2 \times 10^{14} \mathrm{~cm}^{-2}$ range). The results are summarized in Table 4 . The range of values of the derived densities and temperatures from the $\mathrm{HCO}^{+} \mathrm{LVG}$ analysis was used to perform the same analysis with CS for HH 7-11 and HH 34 E, using as completely free parameters only the $\mathrm{CS}$ column density and the size of its emission.

$\mathrm{SO}$ and $\mathrm{C}_{3} \mathrm{H}_{2}$ are the only other species for which, for some objects, we observed multiple transitions and collisional rates are available. For the SO (HH 7-11 A and HH 7-11 B) and $\mathrm{C}_{3} \mathrm{H}_{2}$ (HH 7-11 A) observed transitions we used RADEX, a non-LTE molecular radiative transfer code which assumes an isothermal homogeneous medium (Schöier et al. 2005). To find the possible set of solutions for the $\mathrm{SO}$ and $\mathrm{C}_{3} \mathrm{H}_{2}$ we used the three observed transitions as well as their line ratios with the line intensities corrected by the filling factor. The set of best solutions are shown in Table 4.

\subsection{1. $\mathrm{HH} 7-11$}

For all the clumps we find good fits for $\mathrm{HCO}^{+}$column densities ranging from $5 \times 10^{14}$ to $1 \times 10^{15} \mathrm{~cm}^{-2}$. As an example of the LVG modelling, Fig. 6 (top) shows a set of LVG solutions in the $T_{k}-n\left(\mathrm{H}_{2}\right)$ plane for $\mathrm{HH} 7-11 \mathrm{~A}$. The best fit is obtained for a gas density of $\sim 8 \times 10^{4}-3 \times 10^{5} \mathrm{~cm}^{-3}$, a $T_{k}$ of $15-24 \mathrm{~K}$ and a source size of 20"; while for HH 7-11 B we find similar solutions, for $\mathrm{HH} 7-11 \mathrm{X}$ we obtain a higher gas density range and

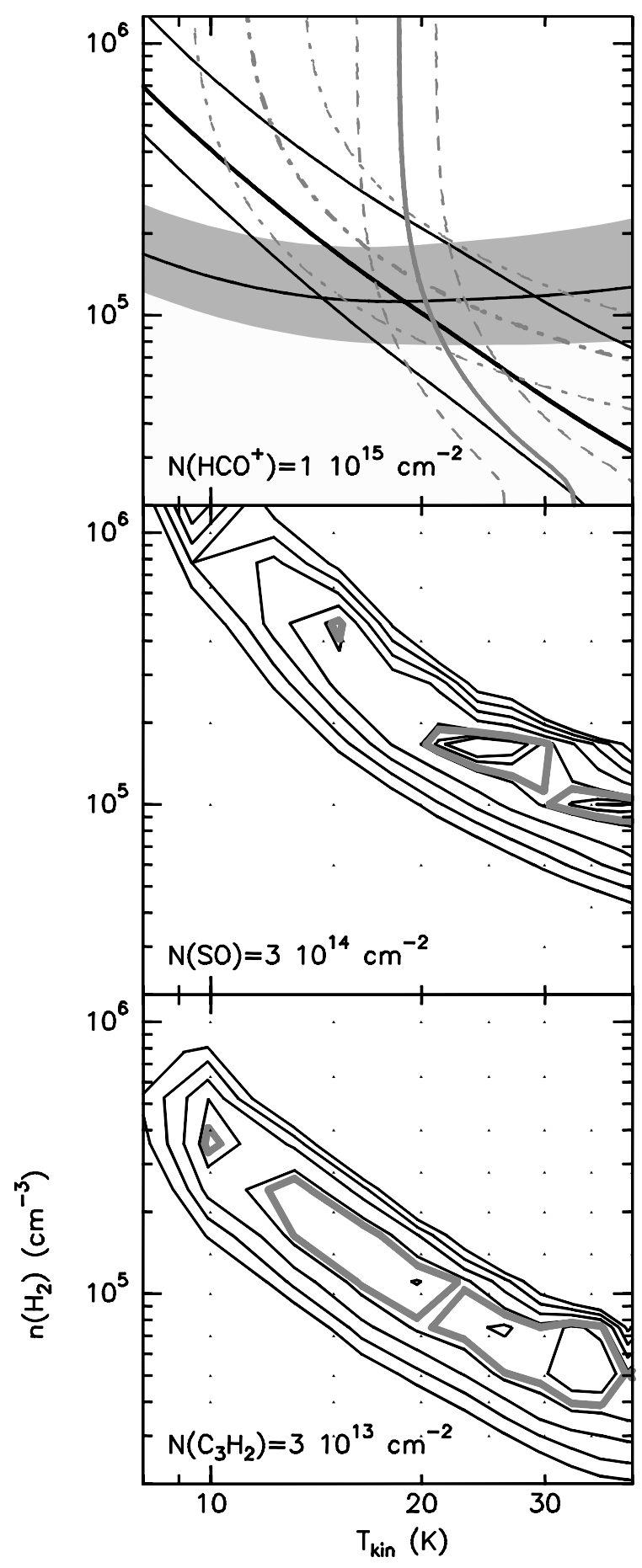

Fig. 6. Plot of the set of LVG and RADEX solutions in the $T_{\mathrm{K}}-n\left(\mathrm{H}_{2}\right)$ plane for HH 7-11 A assuming a source size of $20^{\prime \prime}$ for the following molecules: top panel $\mathrm{HCO}^{+}$, derived using LVG for a column density of $10^{15} \mathrm{~cm}^{-2}$. The grey scale and the thick solid line in its center shows the $\mathrm{HCO}^{+} 3-2$ to $\mathrm{H}^{13} \mathrm{CO}^{+} 3-2$ line ratio. The three solid lines show the $\mathrm{H}^{13} \mathrm{CO}^{+} 1-0$ to $\mathrm{H}^{13} \mathrm{CO}^{+} 3-2$ line ratio. The thick solid and dashed grey line show the the $\mathrm{HCO}^{+} 3-2$ and $\mathrm{HCO}^{+} 3-2$ lines, respectively. Central panel SO, derived using RADEX for a column density of $3 \times 10^{14} \mathrm{~cm}^{-2}$. The thin contours levels are (from lowest to highest contour) $\chi^{2}=20,16,12,8,4,2,1$. The thick grey contour shows the $68 \%$ confidence region of $\chi^{2}$. Bottom panel. $\mathrm{C}_{3} \mathrm{H}_{2}$, derived using RADEX for a column density of $3 \times 10^{13} \mathrm{~cm}^{-2}$. The thin contours levels are (from lowest to highest contour) $\chi^{2}=20,16,12,8,4$. The thick grey contour shows the $68 \%$ confidence region of $\chi^{2}$. 
definitely a lower kinetic temperature. Although HH 7-11 X seems close to $\mathrm{HH} 8$, the low temperature may be an indication that this clump is further away from the highest excitation $\mathrm{HH}$ object. Also note that we do not know the line-of-sight component of the distance between clump and HH object. The derived source size is larger than the estimated size from Rudolph \& Welch (1988) maps. This may be due to the clumps being very close to each other causing their emission to be "contaminated", yielding to an apparently bigger size.

There are two CS transitions detected toward HH 7-11 A, so the LVG analysis have been performed by using as a constrain the lower limit of the kinetic temperature derived from the $\mathrm{LVG} \mathrm{HCO}^{+}$analysis, $T_{k}=15 \mathrm{~K}$. Interestingly, we do not find any solution for a emitting size less than $30^{\prime \prime}$, suggesting that the CS emission is more extended than the $\mathrm{HCO}^{+}$one. Adopting a size of $30^{\prime \prime}$, the set of possible solutions spread out over a large range of volume and column densities. Table 4 shows the CS column density assuming a volume density similar to the value derived from the $\mathrm{HCO}^{+}$.

For HH 7-11 A and X we also performed RADEX analysis of SO and, for $\mathrm{HH}$ 7-11 A, of $\mathrm{C}_{3} \mathrm{H}_{2}$. As for the other molecules we find that the best SO column density fits are reached for values higher than the beam averaged ones. From $\mathrm{SO}$ and $\mathrm{C}_{3} \mathrm{H}_{2}$ we were not able to constrain the temperature of the gas but we notice that it has to be higher than $10 \mathrm{~K}$ (see Fig. 6, central and bottom panel). The derived gas densities are slightly lower than those derived from the $\mathrm{HCO}^{+}$analysis.

\subsection{2. $\mathrm{HH} 1$}

We did not detect the $\mathrm{H}^{13} \mathrm{CO}^{+} 3-2$ line, hence we had to adopt a $3-\sigma$ upper limit for it as well as for the $\left[\mathrm{H}^{12} \mathrm{CO}^{+} 3-\right.$ $2] /\left[\mathrm{H}^{13} \mathrm{CO}^{+} 3-2\right]$ and $\left[\mathrm{H}^{13} \mathrm{CO}^{+} 1-2\right] /\left[\mathrm{H}^{13} \mathrm{CO}^{+} 3-2\right]$ line ratios. We find that, unlike for $\mathrm{HH} 7-11$ clumps, the best fits are obtained for a source size of $10^{\prime \prime}$ consistent with the size found in the literature (Torrelles et al. 1994). This may support the idea that the apparent larger size of the HH 7-11 clumps is merely a consequence of contamination. While the $\mathrm{HCO}^{+}$column and gas density of the $\mathrm{HH} 1$ clump are similar to those for $\mathrm{HH} 7-11$, its temperature is certainly lower and somewhat better constrained.

\subsection{3. $\mathrm{HH} 34$}

We find a remarkably small range of solutions for the kinetic temperature, at 7-11 K for $\mathrm{HH} 34 \mathrm{~A}$ and $8-11 \mathrm{~K}$ for $\mathrm{HH} 34$ E. As for $\mathrm{HH} \mathrm{1,} \mathrm{we} \mathrm{find} \mathrm{that} \mathrm{the} \mathrm{best} \mathrm{solutions} \mathrm{are} \mathrm{obtained}$ for $10^{\prime \prime}-15^{\prime \prime}$ sizes, consistent with those found in the literature (Rudolph et al. 1992). The $\mathrm{HCO}^{+}$column densities range from $5 \times 10^{14}$ to $1 \times 10^{15} \mathrm{~cm}^{-2}$. For HH $34 \mathrm{~A}$ we derive slightly higher gas densities than for HH 34 E. For HH 34 E we also have the two CS transitions, hence we attempted an LVG analysis by fixing the temperature to 10 and $15 \mathrm{~K}$. As in the case of HH 7-11 there are no solutions for emitting sizes smaller than $\sim 30^{\prime \prime}$ and the CS column density is derived assuming a volume density similar to the value derived from the $\mathrm{HCO}^{+}$.

\subsubsection{Summary of LVG analysis}

In summary, the LVG analysis leads to the following preliminary conclusions: $(i)$ The gas densities of the clumps are always higher than those typical of a molecular cloud $\left(\sim 10^{4} \mathrm{~cm}^{-3}\right)$; (ii) $\mathrm{HCO}^{+}$(and CS for $\mathrm{HH} 7-11$ and $\mathrm{HH} 34 \mathrm{E}$ ) column densities derived from LVG are always larger than the beam averaged ones, consistent with the fact that the size of the clumps is smaller than the beam sizes, although in the case of the HH 711 clumps the picture is somewhat more complicated due to the close vicinity of the clumps; (iii) The emitting sizes derived from the LVG analysis of CS seem to be larger than those derived from the $\mathrm{HCO}^{+}$analysis, indicating that the two species possibly arise from different gas components, a result already found by Girart et al. (2005) in their extensive study of the molecular gas ahead of HH 2. (iv) The temperatures are always well constrained and for all the clumps lower than $25 \mathrm{~K}$, with HH7-11 being the warmest. Note that at low temperatures there is no clear pathway for the enhancement of $\mathrm{HCO}^{+}$: we show in VW99 that for $\mathrm{HCO}^{+}$to reach column densities of $\geq 10^{14} \mathrm{~cm}^{-2}$ grain surface reactions and subsequent evaporation of the icy mantles must have occurred, as the main route of formation for $\mathrm{HCO}^{+}$is via the ion-neutral reaction of $\mathrm{C}^{+}$with $\mathrm{H}_{2} \mathrm{O}$. If the temperature is $\leq 100 \mathrm{~K}$, mantle evaporation can only have occurred via photoevaporation. An alternative way might have been if the gas had been shocked in the past reaching higher temperatures and then cooled down. However during the time the gas cools back down to $\sim 10-20 \mathrm{~K}, \mathrm{HCO}^{+}$should be destroyed again. Moreover, we show in VW99 that an enhanced $\mathrm{UV}$ radiation is in any case needed in order to ionize the gas enough to produce large abundances of $\mathrm{C}^{+}$.

\section{Discussion}

As explained in Sect. 2, our main criterion for extending the survey to other molecular species was the strength of $\mathrm{HCO}^{+}$. Table 3 shows that most clumps that exhibit $\mathrm{HCO}^{+}$enhancement also show a rich chemistry, as predicted by the VW99: methanol, when observed, seems to be at least one order of magnitude more abundant than what typically is found in dark molecular clouds or prestellar cores (see Table 5). Of the sulphur species, CS is ubiquitously detected but note however that CS is abundant in dark clouds and hence some of the emission may arise from the surrounding medium rather than the clumps, overall for those clumps with a size much smaller than our beam size. SO and $\mathrm{H}_{2} \mathrm{~S}$ seem to be present in the majority of the clumps where $\mathrm{HCO}^{+}$is enhanced. Of the other sulphur bearing species, OCS, $\mathrm{H}_{2} \mathrm{CS}$, and NS were only observed in HH 7-11 A. We will now analyse our results and look for a possible correlation between the chemical "richness" of a clump, its vicinity to the $\mathrm{HH}$ shock and the strength of the radiation field impinging on the clump.

In this section we attempt a simple analysis of the molecular species detected in our sample. In Sect. 5.1 we summarize the general physical and chemical properties of all the clumps. In Sect. 5.2 we discuss the effects of distance on the chemical processing ahead of $\mathrm{HH}$ objects by looking at the chemistry of different clumps ahead of the same object. However, it is 
Table 5. Fractional abundances with respect to $\mathrm{H}_{2}$ for $\mathrm{HH}$ 7-11 and L1544.

\begin{tabular}{|c|c|c|c|}
\hline \multicolumn{4}{|c|}{ HH 7-11 } \\
\hline Molecule & A & X & L1544 \\
\hline $\mathrm{CO}$ & $3.7(-5)$ & $5.5(-5)$ & $4.9(-6)$ \\
\hline $\mathrm{CH}_{3} \mathrm{OH}$ & $5.0(-9)$ & $9.8(-9)$ & - \\
\hline $\mathrm{HCO}^{+}$ & $2.9(-9)$ & $3.8(-9)$ & $3.9(-10)$ \\
\hline $\mathrm{H}_{2} \mathrm{CO}$ & $1.3(-9)$ & $2.3(-9)$ & - \\
\hline SO & $1.2(-9)$ & $2.0(-9)$ & $4.8(-10)$ \\
\hline $\mathrm{CS}$ & $8.6(-10)$ & $1.5(-9)$ & $8.6(-10)$ \\
\hline $\mathrm{H}_{2} \mathrm{~S}$ & $7.1(-10)$ & $1.1(-9)$ & - \\
\hline $\mathrm{HCN}$ & $3.3(-10)$ & $3.8(-10)$ & $<4(-10)$ \\
\hline OCS & $3.0(-10)$ & - & - \\
\hline $\mathrm{H}_{2} \mathrm{CS}$ & $1.6(-10)$ & - & - \\
\hline $\mathrm{DCO}^{+}$ & $7.1(-11)$ & $8.6(-11)$ & $2.1(-11)$ \\
\hline
\end{tabular}

important to note that the abundance of each molecular species will depend on $i$ ) the gas density; ii) the radiation field; iii) the distance to the source of radiation; $i v$ ) the size of the clump; $v$ ) the age and, to a lesser extent; vi) the kinetic temperature of the gas. Some of these physical parameters have similar effects on the chemistry (e.g. the size and the gas density). Due to such degeneracy it is often difficult to disentangle the effects on the chemistry due to the different parameters. So, for example, for clumps ahead of the same object, where the radiation field is constant, different abundances may not be due solely to the different positioning of the clumps as it may be that the condensations differ in density, size and age. In 5.3 we briefly discuss the properties of the high velocity gas.

\subsection{Physical and chemical properties of clumps ahead of HH objects}

The physical and chemical properties of the gas ahead of the $\mathrm{HH}$ objects are different from those observed in prestellar cores or dark molecular clouds: they are denser than the latter but their small size and the high abundances of certain species, such as $\mathrm{HCO}^{+}$and methanol, make them unlikely candidates for pre-stellar cores. In Table 5, we compare the typical abundances of a pre-stellar core, L1544, with those derived for the HH 7-11 A and X clumps. The abundances of L1544 are taken from Jørgensen et al. (2002), while the HH 7-11 A abundances were derived by dividing the averaged beam column densities by the $\mathrm{H}_{2}$ column density from the dust observations (Hatchell et al. 2005) by assuming a dust temperature of $23 \mathrm{~K}$ (Chini et al. 2001). There are of course uncertainties associated with the derivation of the $\mathrm{H}_{2}$ column densities (and hence of fractional abundances), such as i) the error bars associated with the integrated emission (see Table 10) and ii) the assumption of LTE which affects the molecular abundances estimate. While the first one only leads to a $30 \%$ uncertainty, assuming LTE may lead to a larger uncertainty. The latter is still below the enhancement factor found (i.e $\sim 1$ order of magnitude), although, for some species, the propagation of both errors may become relevant. Although the abundances ahead of HH 7-11 are clearly enhanced with respect to those in L1544, the low abundance of $\mathrm{CO}$ is puzzling: while freeze out on the grains is clearly responsible for its depletion in L1544, in the case of HH 7-11 the UV radiation should have photoevaporated all the ices so no significant amount of CO should be left on the dust unless it remains trapped in a multilayered ice. An alternative possibility is that the condensations we observe are transient and young: if the UV radiation photoevaporated the mantle while depletion was still occurring, there may have been not enough time to form CO. Viti et al. (2003) already observed that, in fact, to match the observations ahead of HH 2 the condensations had to be young and in one of the best matching models, B10, CO has a gas phase abundance of $2 \times 10^{-5}$ at an $A_{\mathrm{V}}$ of $3.5 \mathrm{mag}$ after evaporation. On the other hand it is also possible that the dust column density is overestimated: some of the material may not be associated with the dense clumps and we may be overestimating the amount of dust responsible for the emission, perhaps because we have a too low an opacity. In short, it is hard to know what the absolute abundance of $\mathrm{CO}$ is and no strong conclusion should be drawn from Table 5 beside the fact that clearly some species are enhanced with respect to those in L1544.

An enhancement of species such as $\mathrm{HCO}^{+}, \mathrm{CH}_{3} \mathrm{OH}$ and $\mathrm{SO}$, in absence of shocks, can indeed be an indication of active photochemistry. Jørgensen et al. (2002) do not give a fractional abundance for $\mathrm{CH}_{3} \mathrm{OH}$ for $\mathrm{L} 1544$ but $\mathrm{HCO}^{+}$and $\mathrm{SO}$ are both less abundant by a factor of 5-10, although species such as CS have similar abundances. Interestingly $\mathrm{DCO}^{+}$also seems to be slightly enhanced in $\mathrm{HH} 7-11$ with respect to the value found in $\mathrm{L} 1544$, although the $\mathrm{DCO}^{+} / \mathrm{HCO}^{+}$ratio is consistent with those expected in a dense, low temperature gas (Roberts et al. 2000). In fact a comparison between starless cores and clumps ahead of $\mathrm{HH}$ objects may be misleading in that the anomaly of our clumps is that they are denser than a molecular cloud but much smaller than starless cores. They in fact show similarity with clumps detected by recent high resolution BIMA observations in L673 (Morata et al. 2005) in that they are of similar sizes and they seem to have structures on scales of $\sim 10^{\prime \prime}$ or so but they have different chemistry because they are illuminated by the $\mathrm{HH}$ radiation.

We are unable to compare clumps ahead of other regions with starless cores as we do not know their $\mathrm{H}_{2}$ column density but we compare the column densities across our clumps in Table 3 and Fig. 7.

Before attempting such comparison, it is important to note that, as discussed in Sect. 3 and as shown from our LVG analysis, we are uncertain about the size of all our clumps; hence for some objects, the beam size may be larger than the source size and for species such as $\mathrm{HCO}^{+}$(enhanced only in dense clumps subjected to the radiation) their averaged beam column densities may be a lower limit; however for species that are abundant also in the surrounding medium, such as CS and CO, their beam averaged column densities may be an overestimate of the emission from the clump.

From Table 3 it is clear that all the clumps, except possibly JMG 99 G2, exhibit a similar chemistry: in order to eliminate the uncertainties due to the sizes, let us compare ratios rather than absolute column densities (see Fig. 7); for 


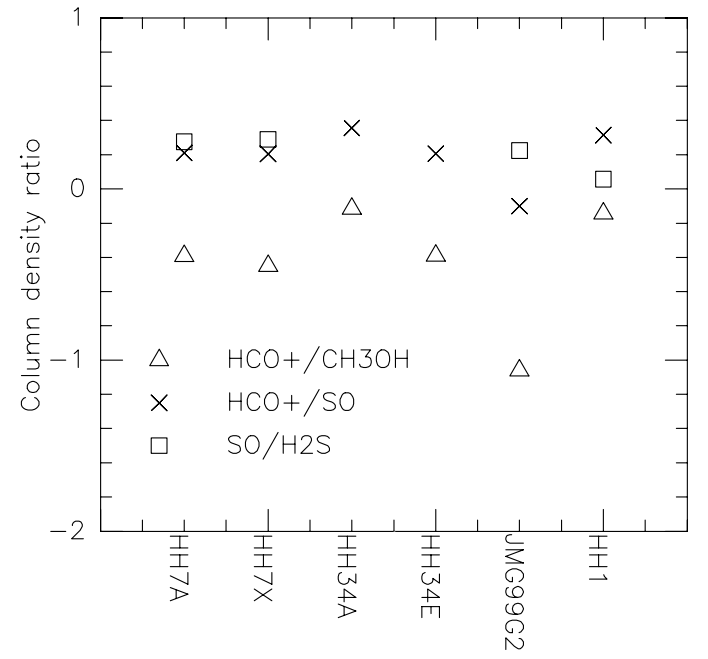

Fig. 7. Ratios (in $\log 10$ scale) of the column densities of selected species for the low velocity gas. HH 7 B, HH 34 C, HH 34 D and JMG 99 G1 are not shown due to lack of data.

example, $\mathrm{HCO}^{+} / \mathrm{CH}_{3} \mathrm{OH}$ is remarkably constant within a factor of 2 for all objects, except for $\mathrm{G} 2$, where it is lower by one order of magnitude. Other ratios, such as $\mathrm{HCO}^{+} / \mathrm{SO}$ or $\mathrm{SO} / \mathrm{H}_{2} \mathrm{~S}$ also show remarkable consistency among the clumps although in G2 the column densities of species characteristic of photochemistry are not as enhanced. In fact, chemically, it appears that while the clumps ahead of $\mathrm{HH} 7-11, \mathrm{HH} 34$ and $\mathrm{HH} 1$ are of the same nature (confirmed also by the column densities derived from the LVG analysis), JMG 99 G1 and G2 have undergone different processing.

Although we do not have an estimate for the temperature of JMG 99 G1 and G2, from our LVG analysis we note that HH 711 A clumps have a higher temperature than $\mathrm{HH} 34$ and $\mathrm{HH} 1$ clumps, therefore $\mathrm{HH} 34$ and $\mathrm{HH} 1$ clumps seem to be the most quiescent, maybe because so far they only have been affected by the UV radiation. Indeed, the line widths of HH 34 are significantly smaller than those of $\mathrm{HH} 7-11\left(\Delta v\left(\mathrm{H}^{13} \mathrm{CO}^{+}\right) \simeq 0.4\right.$ and $1.2 \mathrm{~km} \mathrm{~s}^{-1}$ for HH 34 and $\mathrm{HH} 7-11$, respectively). HH 7 11 clumps, on the other hand, show sign of dynamical interaction with the $\mathrm{HH}$ objects, but such interaction seems not to have affected the chemical nature of the gas yet: the increase in temperature caused by the dynamical interaction is in fact still not sufficient to produce any shock chemistry and hence the chemical properties of $\mathrm{HH} 7-11$ are very similar to those of HH 34 and HH 1 clumps.

Note that, as also explained in Viti et al. (2004), the density enhancements we see ahead of HH 7-11 could not be produced by wind compression as the timescales would be too short for substantial freeze out to have occurred; as described in VW99 and Viti et al. (2003) depletion and subsequent hydrogenation on the grains must have occurred during the formation of the density enhancements, before the arrival of the $\mathrm{HH}$ object, in order for species such as $\mathrm{HCO}^{+}$and $\mathrm{CH}_{3} \mathrm{OH}$ to have formed.
It is tempting to draw an evolutionary scenario around these three different situations with $\mathrm{HH} 34$ and $\mathrm{HH} 1$ clumps being the youngest and JMG $99 \mathrm{G} 1$ and G2 being the oldest clumps. However, it is impossible to disentangle the effects of age, distance from the $\mathrm{HH}$ objects, and radiation, as discussed further in the next section.

Despite the similar chemistry of most of the clumps and the uncertainties with the emission sizes, the (albeit small) differences in column densities (Table 3) may be significant. The three clumps ahead of HH 7-11 may be affected by several HH objects (Fig. 1), hence it is impossible to determine which clump is the most irradiated; however, irradiation from several $\mathrm{HH}$ objects is consistent with the fact that $\mathrm{HH}$ 7-11 clumps show a richer chemistry than others.

Although we do not here attempt any modelling of these clumps, it is interesting to compare the abundances of our clumps with the models computed in Viti et al. (2003). Note that these were detailed models of a particular clump ahead of $\mathrm{HH} 2$ hence we are not looking here for a perfect match. Viti et al. (2003) computed a large grid of models where both the radiation field and densities were varied. Although from the column densities in Table 3 it seems that $\mathrm{HCO}^{+}$is higher ahead of HH 7-11 than ahead of HH 34 and NGC 2264, from our LVG analysis it seems that the densities ahead of HH $7-$ 11 are either lower or the same as those ahead of HH 34 and $\mathrm{HH} 1$. Hence we may expect either that the radiation field from HH 7-11 is higher, or that clumps ahead of HH 7-11 are younger. Figures 3 to 7 in Viti et al. (2003) show the column densities for $\mathrm{HCO}^{+}, \mathrm{CH}_{3} \mathrm{OH}, \mathrm{SO}$ and $\mathrm{H}_{2} \mathrm{CO}$ as a function of time for an $A_{\mathrm{V}} \sim 3$ mag. We compare the column densities in these figures with those derived from our observations here for $\mathrm{HH} \mathrm{7-11} \mathrm{and} \mathrm{find} \mathrm{that} \mathrm{the} \mathrm{best} \mathrm{fitting} \mathrm{models} \mathrm{for} \mathrm{HCO}^{+}$, $\mathrm{CH}_{3} \mathrm{OH}$ and $\mathrm{SO}$ are $\mathrm{B} 7$ and $\mathrm{B} 8$ which have a high radiation field of 1000 Habing and a density of $3 \times 10^{5} \mathrm{~cm}^{-3}$. The abundances of $\mathrm{HCO}^{+}, \mathrm{CH}_{3} \mathrm{OH}$ and $\mathrm{SO}$ may be less ahead of $\mathrm{HH}$ 34 by as much as 1 order of magnitude: although not shown in Viti et al. (2003) we compared two models from their grid where the density $\left(3 \times 10^{5} \mathrm{~cm}^{-3}\right)$, age $(\leq 100 \mathrm{yr})$ and visual extinctions ( $\sim 3 \mathrm{mag})$ were the same but the radiation field varied from 20 to 1000 Habing and we found that $\mathrm{HCO}^{+}$went from $7 \times 10^{12}$ to $5 \times 10^{13} \mathrm{~cm}^{-2}$, SO from $5 \times 10^{14}$ to $2 \times 10^{15}$ and $\mathrm{CH}_{3} \mathrm{OH}$ from $1 \times 10^{14}$ to $6 \times 10^{14} \mathrm{~cm}^{-2}$. However, at much later ages, a higher radiation field implies in fact a lower abundances of these species as the radiation field enhances but also destroys the gas: as explained in VW99 the effect of the irradiation is in fact to create a "wave" of molecular formation where the abundances rise to enhanced values with respect to standard dark cloud chemistry but then fall again as photodissociation begins to dominate. It is difficult therefore to disentangle the effects of radiation, from those of age.

Finally, it is interesting to note that in $\mathrm{HH} \mathrm{7-11} \mathrm{A,} \mathrm{the}$ abundance of $\mathrm{HCS}^{+}$is at least one order of magnitude lower than that of $\mathrm{H}_{2} \mathrm{CS}$. Again, from the Viti et al. 2003 models we find that, as long as the density is greater than $10^{5} \mathrm{~cm}^{-3}$ then $\mathrm{H}_{2} \mathrm{CS} / \mathrm{HCS}^{+}$is always $\geq 10$; but lower radiation fields ( 20 Habing) yield to very high ratios (100-1000) while if $\chi \sim 1000$ Habing the ratio is $\sim 10-50$. 


\subsection{Effects of distance on chemical processing ahead of $\mathrm{HH}$ objects}

In this section we attempt to single out the effect of the distance from other parameters under the assumption that the rich chemistry is mainly due to the UV impinging on the clumps. We therefore compare column densities among clumps belonging to the same region, where not only the radiation field from the $\mathrm{HH}$ shock is constant, but also where we assumed that the size of each clump is the same. In particular HH 34 may be the best region for this analysis as i) the four clumps show some chemical diversity and ii) unlike the HH 7-11 region there seems to be only one $\mathrm{HH}$ object impinging on the clumps.

In the $\mathrm{HH} 34$ region $\mathrm{HH} 34 \mathrm{C}$ and $\mathrm{E}$ are the closest clumps to the $\mathrm{HH}$ object, with A and D 1.6 and 2 times further away (in the plane of the sky; the true distances from the $\mathrm{HH}$ object are of course unknown). From Table 3, HH $34 \mathrm{C}$ and E clearly show higher $\mathrm{HCO}^{+}$column densities than $\mathrm{A}$ and $\mathrm{D}$, although the $\mathrm{CO}$ and $\mathrm{CS}$ column densities are similar, consistent with exposure to a higher UV field. Of $\mathrm{C}$ and $\mathrm{E}, \mathrm{E}$ has the larger $\mathrm{HCO}^{+}$column density, possibly because it has the higher derived gas density derived from the LVG analysis. Moreover, from Table 3, SO and methanol from HH $34 \mathrm{E}$ have higher column densities than the more distant HH 34 A. From this object, we also detect $\mathrm{C}_{3} \mathrm{H}_{2}$, not usually detected in quiescent dense clumps. This increase in chemical activity where the UV radiation field would be expected to be strongest is consistent with the UV chemistry, though a variation in density or age of the clumps cannot be ruled out as an alternative cause.

\subsection{The high velocity component}

HH 7-11 A and JMG 99 G1 are the positions where the high velocity emission is brighter. We calculated the column density ratio with $\mathrm{CO}$ for several species (from the ${ }^{13} \mathrm{CO}$ spectra), i.e., the molecular fractional abundances with respect to the $\mathrm{CO}$ (see Sect. 4). Figure 8 shows the fractional abundances with respect to the $\mathrm{CO}$ for several molecules as a function of the outflow velocity.

From Fig. 8, it is clear that the $\mathrm{HCO}^{+}$relative abundance of the HH 7-11 high velocity component increases by more than an order of magnitude (a factor of $\sim 30$ ) between the slowest and fastest outflow components. This enhancement is also seen in $\mathrm{H}_{2} \mathrm{CO}$ and $\mathrm{CH}_{3} \mathrm{OH}$ for outflow velocities lower than $\sim 6 \mathrm{~km} \mathrm{~s}^{-1}$ with respect to the systemic velocity. On the other hand, $\mathrm{CS}$ does not change its relative abundance. The $\mathrm{HCO}^{+}$ abundance enhancement at higher outflow velocities was first reported by Girart et al. (1999) towards the NGC 2071 molecular outflow. Recently, Dent et al. (2003) has found the same behaviour for the $\mathrm{HCO}^{+}$towards the high velocity gas around $\mathrm{HH}$ 2. However, $\mathrm{HCO}^{+}$is the only high density molecular tracer detected in the high velocity $\mathrm{HH} 2$ component and this case could be a different scenario from NGC 2071 or HH 711 , since in $\mathrm{HH} 2$ the lack of other molecular high velocity gas other than $\mathrm{HCO}^{+}$can be explained by the presence of strong UV field. In any case the clear enhancement of the $\mathrm{HCO}^{+}$abundance with respect to other molecular tracers with increasing

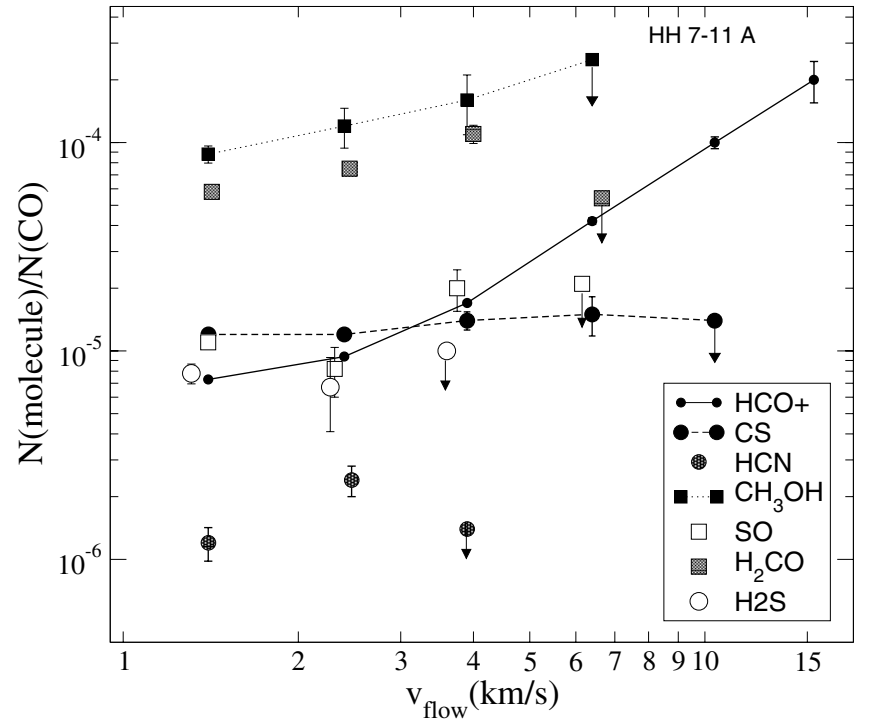

Fig. 8. Fractional abundance with respect to the $\mathrm{CO}$ of the high velocity gas for several molecules toward HH 7-11 A. $v_{\text {flow }}=v_{\mathrm{LSR}}-v_{\mathrm{amb}}$, where $v_{\mathrm{amb}}$ is the systemic velocity of the ambient gas.

high velocity indicates that it traces the molecular outflow well at higher velocities, whatever causes its enhancement.

JMG 99 G1 not only has a spectral feature quite different from the high velocity wings seen in HH 7-11 A but the chemical properties are also very different. In JMG 99 G1 the $\mathrm{CH}_{3} \mathrm{OH}$ is by far the more abundant high density molecular tracer with a $X\left[\mathrm{CH}_{3} \mathrm{OH}\right] / X[\mathrm{CO}] \simeq 2-4 \times 10^{-3}$. This abundance is also found in strongly shock molecular clumps such those in L1157 (Bachiller et al. 2001), and IRAS 21391+5802 (Beltrán et al. 2002). The $\mathrm{HCO}^{+}$on the other hand is almost one order of magnitude lower than in HH 7-11, although its abundance also increases with the outflow velocity. Even the spectral shape of the molecular emission in G1 is similar to the spectral shape of the lines detected in the L1157 clumps: Most of the lines shown are blueshifted (by 2-3 $\mathrm{km} \mathrm{s}^{-1}$ with respect to the cloud velocity) and are significantly broader $\left(\Delta v \simeq 4-5 \mathrm{~km} \mathrm{~s}^{-1}\right)$ than the lines from the other positions. Such emission may in fact come from clumps similar to those seen along the L1157 and CB3 outflows (Bachiller et al. 2001; Codella et al. 1999): Viti et al. (2004) found that such clumpiness may be a consequence of a pre-existing density enhancement and of its interaction with the outflow.

\section{Conclusions}

We have performed a survey of molecular species ahead of several $\mathrm{HH}$ objects, where enhanced $\mathrm{HCO}^{+}$emission was previously observed. Recent modelling have supported the idea that such enhancement is due to $\mathrm{UV}$ radiation from the $\mathrm{HH}$ object lifting the ice mantles from the grains in the clumpy medium ahead of the jet, which then would drive a photochemistry. If this model is correct then other molecular species (such as $\mathrm{CH}_{3} \mathrm{OH}$, SO etc) should also be enhanced. A recent molecular survey of the gas ahead of HH 2 seems to support such model (Girart et al. 2005). The aims of this present survey were to investigate: 1) whether the rich chemistry observed ahead of 
HH 2, and predicted by the VW99 model, was indeed characteristic of the gas ahead of all Herbig-Haro objects; 2) the chemical differentiation that may arise among clumps ahead of different objects, which may differ in radiation field strengths.

We have selected $4 \mathrm{HH}$ objects regions: in some of these regions enhanced $\mathrm{HCO}^{+}$emission had been detected from several clumps. Hence, in total we observed ten positions. For each position we searched for more than one species and, if possible, for more than one transition per species. We find that, chemically, the richest gas was that ahead of the HH 7-11 complex, in particular at the HH 7-11 A position. In some regions we also detected a high velocity gas component. Beside estimating the averaged beam column densities for all the species observed, we have performed, where possible, LVG analyses to constrain the physical properties of the gas. The main conclusions from our LVG analysis are:

1. The gas densities of all the clumps observed are always higher than those typical of molecular clouds by at least a factor of 5, but probably higher.

2. The derived gas temperatures are alway quite low $(<15 \mathrm{~K})$ apart for the clumps ahead of $\mathrm{HH} 7-11$ where the maximum temperature does not in any case exceed $25 \mathrm{~K}$.

3. $\mathrm{HCO}^{+}, \mathrm{SO}$ and $\mathrm{CS}$ column densities derived from our LVG and RADEX analyses are higher than the beam averaged ones. This indicates that the size of the emitting region is smaller than the beam size.

4. The emitting size derived from the LVG analysis of CS are larger than those estimated from the $\mathrm{HCO}^{+}$analysis. It may be that these two species arise from different gas components, consistent with previous studies (Girart et al. 2005). In addition, the $\mathrm{HCO}^{+}$abundance is enhanced with respect to the starless core in L1544, whereas the CS has similar abundances. All this suggests that at least a fraction of the CS emission may arise from a cloud component or clumps not illuminated by he $\mathrm{HH}$ objects.

By comparing column densities among the several clumps we also attempt a very simple chemical analysis in light of the photochemical model. We find that the abundances of the observed species ahead of the surveyed $\mathrm{HH}$ objects are higher than those found in a typical starless core and that by comparing the column densities among the different clumps we find that the clumps ahead of HH 7-11, HH 34 and HH 1 are of the same chemical nature, supporting the idea that the gas ahead of all $\mathrm{HH}$ objects exhibit a rich chemistry.

Acknowledgements. S.V. and J.H. both acknowledge individual financial support from a PPARC Advanced Fellowship. J.M.G. acknowledges support by SEUI grant AYA2002-00205. S.V. and J.M.G. acknowledge support by a joined RS and CSIC travel grant. We thank the staff of the IRAM $30 \mathrm{~m}$ telescope for help with the observations.

\section{References}

Bachiller, R., Pérez Gutiérrez, M., Kumar, M. S. N., \& Tafalla, M. 2001, A\&A, 372
Beltrán, M. T., Girart, J. M., Estalella, R., Ho, P. T. P., \& Palau, A. 2002, ApJ, 573, 246

Caselli, P., Walmsley, C. M., Terzieva, R., \& Herbst, E. 1998, ApJ, 499, 234

Cernis, K. 1990, Ap\&SS, 166, 315

Chini, R., Ward-Thompson, D., Kirk, J. M., et al. 2001, A\&A, 369, 155

Codella, C., \& Bachiller, R. 1999, A\&A, 350, 659

Davis, C. J., Dent, W. R. F., \& Burnell, S. J. B. 1990, MNRAS, 244, 173

Dent, W. R. F., Furuya, R. S., \& Davis, C. J. 2003, MNRAS, 339, 633

Dent, W. R. F., Cunningham, C., Hayward, R., et al. 1993, MNRAS, 262, L13

Evans, N. J. II, Rawlings, J. C. M., Shirley, Y. L., \& Mundy, L. G. 2001, ApJ, 557, 193

Herbig, G. H., \& Jones, B. F. 1981, AJ, 86, 1232

Garay, G., Mardones, D., Rodríguez, L. F., Caselli, P. \& Bourke, T. L. 2002, ApJ, 567980

Girart, J. M., Rodriguez, L. F., Anglada, G., et al. 1994, ApJ, 435, L145

Girart, J. M., Ho, P. T. P., Rudolph, A. L., et al. 1999, ApJ, 522, 921

Girart, J. M., Estalella, R., Ho, P. T. P., \& Rudolph, A. L. 2000, ApJ, 539, 763

Girart, J. M., Viti, S., Williams, D. A., Estalella, R., \& Ho, P. T. P. 2002, A\&A, 388, 1004

Girart, J. M., Viti, S., Estalella, R., \& Williams, D. A. 2005, A\&A, 439,601

Gómez, J. F., Curiel, S., Torrelles, J. M., et al. 1994, ApJ, 436, 749

Hatchell, J., Richer, J., Fuller, G. A., et al. 2005, A\&A, 440, 151

Jørgensen, J. K., Schöier, F. L., \& van Dishoeck, E. F. 2002, A\&A, 389,908

Lada, C. J., \& Fich, M. 1996, ApJ, 459, 638

Lefloch, B., Cernicharo, J., Cabrit, S., \& Cesarsky, D. 2005, A\&A, 433, 217

Mehringer, D. M. 1996, ApJ, 462, 355

Morata, O., Girart, J. M., \& Estalella, R. 2005, A\&A, 435, 113

Pravdo, S. H., Rodriguez, L. F., Curiel, S., et al. 1985, ApJ, 293, L35

Reipurth, B., Heathcote, S., Morse, J., Hartigan, P., \& Bally, J. 2002, AJ, 123, 362

Roberts, H., \& Millar, T. J. 2000, A\&A, 361, 388

Rodríguez, L. F., Anglada, G., \& Curiel, S. 1999, ApJS, 125, 427

Rudolph, A. L., Bachiller, R., Rieu, N. Q., et al. 2001, ApJ, 558, 204

Rudolph, A., \& Welch, W. J. 1988, ApJ, 326, L31

Rudolph, A., \& Welch, W. J. 1992, ApJ, 395, 488

Schöier, F. L., van der Tak, F. F. S., van Dishoeck, E. F., \& Black, J. H. 2005, A\&A, 432, 369

Solf, J., \& Boehm, K. H. 1987, AJ, 93, 1172

Torrelles, J. M., Rodriguez, L. F., Canto, J., et al. 1992, ApJ, 396, L95

Torrelles, J. M., Gomez, J. F., Ho, P. T. P., et al. 1993, ApJ, 417, 655

Torrelles, J. M., Gomez, J. F., Ho, P. T. P., et al. 1994, ApJ, 435, 290

Viti, S., \& Williams, D. A. 1999, MNRAS, 310, 517 (VW99)

Viti, S., Girart, J. M., Garrod, R., Williams, D. A., \& Estalella, R. 2003, A\&A, 399, 187

Viti, S., Codella, C., Benedettini, M., \& Bachiller, R. 2004, MNRAS, 350,1029

Ward-Thompson, D., Eiroa, C., \& Casali, M. M. 1995, MNRAS, 273, L25 
S. Viti et al.: A molecular survey ahead of $\mathrm{HH}$ objects, Online Material $p 1$

\section{Online Material}


S. Viti et al.: A molecular survey ahead of HH objects, Online Material p 3

Table 7. Molecular line results for HH 34 E \& A.

\begin{tabular}{|c|c|c|c|c|c|c|c|c|}
\hline \multirow[b]{2}{*}{$\begin{array}{l}\text { Molecular } \\
\text { Transition }\end{array}$} & \multicolumn{4}{|c|}{ HH 34 A } & \multicolumn{4}{|c|}{ HH $34 \mathrm{E}$} \\
\hline & $\begin{array}{l}T_{\mathrm{MB}} \\
(\mathrm{K})\end{array}$ & $\begin{array}{r}\int T_{\mathrm{MB}} \mathrm{d} v \\
\left(\mathrm{~K} \mathrm{~km} \mathrm{~s}^{-1}\right)\end{array}$ & $\begin{array}{c}v_{\mathrm{LSR}} \\
\left(\mathrm{km} \mathrm{s}^{-1}\right)\end{array}$ & $\begin{array}{r}\Delta v_{\mathrm{LSR}} \\
\left(\mathrm{km} \mathrm{s}^{-1}\right)\end{array}$ & $\begin{array}{l}T_{\mathrm{MB}} \\
(\mathrm{K})\end{array}$ & $\begin{array}{r}\int T_{\mathrm{MB}} \mathrm{d} v \\
\left(\mathrm{~K} \mathrm{~km} \mathrm{~s}^{-1}\right)\end{array}$ & $\begin{array}{c}v_{\mathrm{LSR}} \\
\left(\mathrm{km} \mathrm{s}^{-1}\right)\end{array}$ & $\begin{array}{r}\Delta v_{\mathrm{LSR}} \\
\left(\mathrm{km} \mathrm{s}^{-1}\right)\end{array}$ \\
\hline${ }^{13} \mathrm{CO} 2-1^{a}$ & $11.7(3)$ & $10.04(2)$ & $8.60(9)$ & $0.65(9)$ & $12.2(3)$ & $11.15(8)$ & $8.7(1)$ & $0.6(1)$ \\
\hline $\mathrm{C}^{18} \mathrm{O} 2-1^{a}$ & $5.51(15)$ & $2.70(4)$ & $8.70(1)$ & $0.46(1)$ & $4.2(2)$ & $2.65(8)$ & $8.73(1)$ & $0.64(2)$ \\
\hline $\mathrm{HCO}^{+} 1-0$ & $4.06(9)$ & $2.31(3)$ & $8.49(1)$ & $0.54(1)$ & $2.85(12)$ & $2.00(5)$ & $8.49(1)$ & $0.66(2)$ \\
\hline $\mathrm{HCO}^{+} 3-2$ & $2.3(4)$ & $1.14(13)$ & $8.51(3)$ & $0.46(5)$ & $2.4(5)$ & $1.69(19)$ & $8.63(4)$ & $0.65(10)$ \\
\hline $\mathrm{H}^{13} \mathrm{CO}^{+} 1-0$ & $0.72(8)$ & $0.31(2)$ & $8.63(1)$ & $0.40(3)$ & $0.91(7)$ & $0.44(2)$ & $8.78(1)$ & $0.46(2)$ \\
\hline $\mathrm{H}^{13} \mathrm{CO}^{+} 3-2$ & $0.6(2)$ & $0.20(6)$ & $8.3(1)$ & $0.3(1)$ & $\lesssim 1.0$ & $\lesssim 0.22$ & & \\
\hline $\mathrm{DCO}^{+} 2-1$ & $1.2(2)$ & $0.46(3)$ & $8.54(1)$ & $0.37(3)$ & $1.25(15)$ & $0.55(3)$ & $8.69(1)$ & $0.42(2)$ \\
\hline $\mathrm{DCO}^{+} 3-2$ & $0.7(2)$ & $0.26(4)$ & $8.55(4)$ & $0.37^{b}$ & $0.67(16)$ & $0.20(4)$ & $8.43(5)$ & $0.3(1)$ \\
\hline $\mathrm{HCN} 3-2$ & $\lesssim 0.75$ & $\lesssim 0.20$ & & & $\lesssim 0.75$ & $\lesssim 0.20$ & & \\
\hline $\mathrm{H}_{2} \mathrm{CO} 2_{0,2}-1_{0,1}$ & $1.9(2)$ & $0.76(5)$ & $8.48(1)$ & $0.38(4)$ & $1.5(3)$ & $0.86(12)$ & $8.56(3)$ & $0.56(10)$ \\
\hline $\mathrm{H}_{2} \mathrm{CO} 3_{1,3}-2_{1,2}$ & & & & & $1.43(11)$ & $0.87(3)$ & $8.78(1)$ & $0.57(2)$ \\
\hline $\mathrm{CH}_{3} \mathrm{OH} 2_{0}-1_{0} \mathrm{~A}^{+}$ & $0.68(7)$ & $0.31(2)$ & $8.64(1)$ & $0.43(2)$ & $1.41(7)$ & $0.68(2)$ & $8.75(1)$ & $0.42(1)$ \\
\hline $\mathrm{CH}_{3} \mathrm{OH} 2_{-1}-1_{-1} \mathrm{E}$ & $0.42(7)$ & $0.19(2)$ & fixed & fixed & $1.12(7)$ & $0.54(2)$ & fixed & fixed \\
\hline $\mathrm{CH}_{3} \mathrm{OH} 2_{0}-1_{0} \mathrm{E}$ & $\lesssim 0.21$ & $\lesssim 0.06$ & & & $\lesssim 0.21$ & $\lessgtr 00.06$ & & \\
\hline $\mathrm{CH}_{3} \mathrm{OH} 3_{0}-2_{0} \mathrm{~A}^{+}$ & $\lessgtr 0.21$ & $\lessgtr 0.06$ & & & $1.6(2)$ & $0.60(3)$ & $8.79(1)$ & $0.35(1)$ \\
\hline $\mathrm{CH}_{3} \mathrm{OH} 3_{-1}-2_{-1} \mathrm{E}$ & $\lesssim 0.21$ & $\lesssim 0.06$ & & & $1.6(2)$ & $0.58(3)$ & fixed & fixed \\
\hline CS 3-2 & $2.5(3)$ & $1.37(6)$ & $8.58(1)$ & $0.52(2)$ & $2.4(3)$ & $1.65(8)$ & $8.57(2)$ & $0.66(3)$ \\
\hline CS 5-4 & & & & & $0.5(2)$ & $0.17(3)$ & $8.75(3)$ & $0.35(5)$ \\
\hline $\mathrm{SO} 3_{2}-2_{1}$ & $1.60(7)$ & $0.80(2)$ & $8.72(1)$ & $0.45(1)$ & $3.18(8)$ & $1.63(2)$ & $8.76(1)$ & $0.48(1)$ \\
\hline $\mathrm{SO} 6_{5}-5_{4}$ & $\lesssim 1.2$ & $\lessgtr 0.26$ & & & $\lesssim 1.2$ & $\lessgtr 0.26$ & & \\
\hline $\mathrm{SO} 7_{6}-6_{5}$ & $\lesssim 2.5$ & $\lesssim 0.68$ & & & $\lesssim 2.5$ & $\lesssim 0.55$ & & \\
\hline $\mathrm{C}_{3} \mathrm{H}_{2} 2_{1,2}-1_{0,1}$ & & & & & $0.41(6)$ & $0.17(2)$ & $8.78(2)$ & $0.39(4)$ \\
\hline $\mathrm{C}_{3} \mathrm{H}_{4} 5_{0}-4_{0}$ & & & & & $\lesssim 0.19$ & $\lesssim 0.05$ & & \\
\hline $\mathrm{H}_{2} \mathrm{CS} 4_{0,4}-3_{0,3}$ & & & & & $\lessgtr 0.36$ & $\$ 0.09$ & & \\
\hline NS 7/2-5/2 161.297 & & & & & $\lesssim 0.45$ & $\lesssim 0.11$ & & \\
\hline
\end{tabular}

${ }^{a}$ The ${ }^{13} \mathrm{CO} 1-0$ is non-Gaussian. Line parameters derived from the zero (integrated intensity), first (line velocity) and second (line width) order moments of the emission in the 8.30-9.20 $v_{\text {LSR }}$ range (the velocity range of $\mathrm{C}^{18} \mathrm{O}$ emission).

${ }^{b}$ Weak line: fixed line width with the value of the $\mathrm{DCO}^{+} 2-1$ line.

Table 8. Molecular line results for HH 34 C \& D.

\begin{tabular}{|c|c|c|c|c|c|c|c|c|}
\hline \multirow[b]{2}{*}{$\begin{array}{l}\text { Molecular } \\
\text { transition }\end{array}$} & \multicolumn{4}{|c|}{ HH $34 \mathrm{C}$} & \multicolumn{4}{|c|}{ HH 34 D } \\
\hline & $\begin{array}{l}T_{\mathrm{MB}} \\
(\mathrm{K})\end{array}$ & $\begin{array}{r}\int T_{\mathrm{MB}} \mathrm{d} v \\
\left(\mathrm{~K} \mathrm{~km} \mathrm{~s}^{-1}\right)\end{array}$ & $\begin{array}{c}v_{\mathrm{LSR}} \\
\left(\mathrm{km} \mathrm{s}^{-1}\right)\end{array}$ & $\begin{array}{r}\Delta v_{\mathrm{LSR}} \\
\left(\mathrm{km} \mathrm{s}^{-1}\right)\end{array}$ & $\begin{array}{l}T_{\mathrm{MB}} \\
(\mathrm{K})\end{array}$ & $\begin{array}{r}\int T_{\mathrm{MB}} \mathrm{d} v \\
\left(\mathrm{~K} \mathrm{~km} \mathrm{~s}^{-1}\right)\end{array}$ & $\begin{array}{c}v_{\mathrm{LSR}} \\
\left(\mathrm{km} \mathrm{s}^{-1}\right)\end{array}$ & $\begin{array}{r}\Delta v_{\mathrm{LSR}} \\
\left(\mathrm{km} \mathrm{s}^{-1}\right)\end{array}$ \\
\hline${ }^{13} \mathrm{CO} 2-1^{a}$ & $11.2(3)$ & $10.30(7)$ & $8.53(9)$ & $0.7(1)$ & $11.3(3)$ & $9.70(7)$ & $8.55(9)$ & $0.7(1)$ \\
\hline $\mathrm{HCO}^{+} 1-0$ & $3.79(9)$ & $2.95(2)$ & $8.51(1)$ & $0.73(1)$ & $2.78(9)$ & $2.22(3)$ & $8.57(1)$ & $0.75(1)$ \\
\hline $\mathrm{HCO}^{+} 3-2$ & $2.9(5)$ & $1.6(2)$ & $8.53(3)$ & $0.51(9)$ & $1.5(4)$ & $0.93(16)$ & $8.64(5)$ & $0.60(11)$ \\
\hline CS 3-2 & $2.3(3)$ & $1.25(5)$ & $8.57(1)$ & $0.52(3)$ & $2.0(3)$ & $1.14(6)$ & $8.59(1)$ & $0.55(4)$ \\
\hline
\end{tabular}

${ }^{a}$ The ${ }^{13} \mathrm{CO} 1-0$ is non-Gaussian. Line parameters derived from the zero (integrated intensity), first (line velocity) and second (line width) order moments of the emission in the 8.0-9.0 $\mathrm{v}_{\mathrm{LSR}}$ range (the velocity range of $\mathrm{HCO}^{+}$emission). 
Table 9. Molecular line results for HH 1.

\begin{tabular}{|c|c|c|c|c|}
\hline $\begin{array}{l}\text { Molecular } \\
\text { transition }\end{array}$ & $\begin{array}{l}T_{\mathrm{MB}} \\
(\mathrm{K})\end{array}$ & $\begin{array}{r}\int T_{\mathrm{MB}} \mathrm{d} v \\
\left(\mathrm{~K} \mathrm{~km} \mathrm{~s}^{-1}\right)\end{array}$ & $\begin{array}{c}v_{\mathrm{LSR}} \\
\left(\mathrm{km} \mathrm{s}^{-1}\right)\end{array}$ & $\begin{array}{r}\Delta v_{\mathrm{LSR}} \\
\left(\mathrm{km} \mathrm{s}^{-1}\right)\end{array}$ \\
\hline${ }^{13} \mathrm{CO} 2-1^{a}$ & $16.7(3)$ & $29.7(2)$ & $9.1(1)$ & $1.6(2)$ \\
\hline $\mathrm{C}^{18} \mathrm{O} 2-1^{a}$ & $5.60(9)$ & $6.25(5)$ & $9.1(1)$ & $1.3(1)$ \\
\hline $\mathrm{HCO}^{+} 1-0$ & $3.21(10)$ & $7.10(6)$ & $9.21(1)$ & $2.07(2)$ \\
\hline $\mathrm{HCO}^{+} 3-2$ & $1.6(5)$ & $2.50(29)$ & $9.12(8)$ & $1.45(20)$ \\
\hline $\mathrm{H}^{13} \mathrm{CO}^{+} 1-0$ & $0.45(5)$ & $0.42(2)$ & $9.31(2)$ & $0.87(5)$ \\
\hline $\mathrm{H}^{13} \mathrm{CO}^{+} 3-2$ & $\lessgtr 0.33$ & $\lessgtr 0.15$ & & \\
\hline $\mathrm{DCO}^{+} 2-1$ & $0.33(9)$ & $0.18(3)$ & $9.19(4)$ & $0.57(13)$ \\
\hline $\mathrm{DCO}^{+} 3-2$ & $0.26(10)$ & $0.13(3)$ & $9.18(7)$ & $0.45(13)$ \\
\hline HCN 3-2 & $0.35(17)$ & $0.56(11)$ & $9.14(16)$ & $1.5(3)$ \\
\hline $\mathrm{H}_{2} \mathrm{CO} 2_{0,2}-1_{0,1}$ & $0.8(2)$ & $1.2(2)$ & $9.18(9)$ & $1.5(3)$ \\
\hline $\mathrm{CH}_{3} \mathrm{OH} 2_{0}-1_{0} \mathrm{~A}^{+}$ & $0.23(5)$ & $0.61(7)$ & $9.20(10)$ & $2.4(2)$ \\
\hline $\mathrm{CH}_{3} \mathrm{OH} 2_{-1}-1_{-1} \mathrm{E}$ & $0.20(5)$ & $0.54(7)$ & fixed & fixed \\
\hline $\mathrm{CH}_{3} \mathrm{OH} 2_{0}-1_{0} \mathrm{E}$ & $\lesssim 0.18$ & $\lesssim 0.13$ & & \\
\hline $\mathrm{CH}_{3} \mathrm{OH} 3_{0}-2_{0} \mathrm{~A}^{+}$ & $\lesssim 0.21$ & $\lesssim 0.11$ & & \\
\hline CS 3-2 & $1.85(16)$ & $2.03(11)$ & $9.09(3)$ & $1.03(8)$ \\
\hline $\mathrm{SO} 3_{2}-2_{1}$ & $0.84(3)$ & $1.70(2)$ & $9.05(1)$ & $1.92(3)$ \\
\hline SO $6_{5}-5_{4}$ & $\lesssim 0.6$ & $\lesssim 0.28$ & & \\
\hline $\mathrm{SO} 7_{6}-6_{5}$ & $\$ 2.0$ & $\lesssim 0.93$ & & \\
\hline $\mathrm{H}_{2} \mathrm{~S} 1_{1,0}-0_{0,1}$ & $1.3(3)$ & $0.98(15)$ & $9.01(6)$ & $0.73(14)$ \\
\hline
\end{tabular}

${ }^{a}$ The ${ }^{13} \mathrm{CO} 1-0, \mathrm{C}^{18} \mathrm{O} 1$ - lines are non-Gaussian. Line parameters derived from the zero (integrated intensity), first (line velocity) and second (line width) order moments of the emission in the 8.10-10.30 $v_{\mathrm{LSR}}$ range (the velocity range of $\mathrm{HCO}^{+}$emission). 
S. Viti et al.: A molecular survey ahead of HH objects, Online Material p 5

Table 10. Molecular line results for NGC 2264G.

\begin{tabular}{|c|c|c|c|c|c|c|c|c|}
\hline \multirow[b]{2}{*}{$\begin{array}{l}\text { Molecular } \\
\text { transition }\end{array}$} & \multicolumn{4}{|c|}{ JMG1 } & \multicolumn{4}{|c|}{ JMG2 } \\
\hline & $\begin{array}{l}T_{\mathrm{MB}} \\
(\mathrm{K})\end{array}$ & $\begin{array}{r}\int T_{\mathrm{MB}} \mathrm{d} v \\
\left(\mathrm{~K} \mathrm{~km} \mathrm{~s}^{-1}\right)\end{array}$ & $\begin{array}{c}v_{\mathrm{LSR}} \\
\left(\mathrm{km} \mathrm{s}^{-1}\right)\end{array}$ & $\begin{array}{r}\Delta v_{\mathrm{LSR}} \\
\left(\mathrm{km} \mathrm{s}^{-1}\right)\end{array}$ & $\begin{array}{l}T_{\mathrm{MB}} \\
(\mathrm{K})\end{array}$ & $\begin{array}{r}\int T_{\mathrm{MB}} \mathrm{d} v \\
\left(\mathrm{~K} \mathrm{~km} \mathrm{~s}^{-1}\right)\end{array}$ & $\begin{array}{c}v_{\mathrm{LSR}} \\
\left(\mathrm{km} \mathrm{s}^{-1}\right)\end{array}$ & $\begin{array}{r}\Delta v_{\mathrm{LSR}} \\
\left(\mathrm{km} \mathrm{s}^{-1}\right)\end{array}$ \\
\hline \multirow[t]{3}{*}{${ }^{13} \mathrm{CO} 2-1^{0}$} & $1.0(2)$ & $1.04(12)$ & fixed & fixed & $2.5(3)$ & $2.46(7)$ & $4.86(8)$ & $0.76(9)$ \\
\hline & $1.7(2)$ & $8.5(3)$ & $3.42(10)$ & $4.7(2)$ & $2.5(3)$ & $2.46(7)$ & $4.86(8)$ & $0.76(9)$ \\
\hline & $1.4(2)$ & $1.39(12)$ & $8.63(4)$ & $0.97(9)$ & $2.5(3)$ & $2.46(7)$ & $4.86(8)$ & $0.76(9)$ \\
\hline $\mathrm{C}^{18} \mathrm{O} 2-1$ & $0.61(18)$ & $0.65(9)$ & $4.83(7)$ & $1.01(18)$ & $1.04(19)$ & $0.86(8)$ & $4.83(4)$ & $0.77(9)$ \\
\hline $\mathrm{HCO}^{+} 1-0^{a}$ & $0.7(1)$ & $2.30(6)$ & $1.6(4)$ & $3.0(5)$ & $0.4(1)$ & $0.33(3)$ & $4.7(2)$ & $0.9(2)$ \\
\hline \multirow[t]{2}{*}{$\mathrm{HCO}^{+} 3-2^{b}$} & $0.7(2)$ & $0.6(2)$ & $4.9(1)$ & $0.9(3)$ & $2.8(4)$ & $2.77(14)$ & $4.6(1)$ & $0.8(1)$ \\
\hline & $0.8(2)$ & $2.2(4)$ & $2.1(2)$ & $2.5(6)$ & $2.8(4)$ & $2.77(14)$ & $4.6(1)$ & $0.8(1)$ \\
\hline $\mathrm{H}^{13} \mathrm{CO}^{+} 1-0^{d}$ & $0.06(3)$ & $0.06(2)$ & $5.1(3)$ & $0.9(5)$ & $0.24(5)$ & $0.20(2)$ & $4.64(4)$ & $0.78(7)$ \\
\hline $\mathrm{H}^{13} \mathrm{CO}^{+} 3-2$ & $\lesssim 0.5$ & $\lessgtr 0.3$ & & & $\lesssim 0.75$ & $\lesssim 0.29$ & & \\
\hline $\mathrm{DCO}^{+} 2-1$ & $\lesssim 0.15$ & $\lesssim 0.07$ & & & $0.15(5)$ & $0.19(3)$ & $4.44(9)$ & $1.1(2)$ \\
\hline $\mathrm{DCO}^{+} 3-2$ & $\lesssim 0.18$ & $\lesssim 0.08$ & & & $\lesssim 0.27$ & $\lesssim 0.08$ & & \\
\hline HCN 3-2 & $0.69(14)$ & $3.87(18)$ & $2.10(12)$ & $5.3(3)$ & $0.84(12)$ & $2.45(14)$ & $4.05(7)$ & $2.7(2)$ \\
\hline $\mathrm{CH}_{3} \mathrm{OH} 2_{0}-1_{0} \mathrm{~A}^{+}$ & $0.48(2)$ & $2.78(5)$ & $2.29(6)$ & $5.40(6)$ & $0.56(3)$ & $1.11(2)$ & $4.43(2)$ & $1.83(3)$ \\
\hline $\mathrm{CH}_{3} \mathrm{OH} 2_{-1}-1_{-1} \mathrm{E}$ & $0.35(2)$ & $2.03(5)$ & fixed & fixed & $0.39(3)$ & $0.75(2)$ & fixed & fixed \\
\hline $\mathrm{CH}_{3} \mathrm{OH} 2_{0}-1_{0} \mathrm{E}$ & $0.09(2)$ & $0.53(5)$ & fixed & fixed & $0.07(3)$ & $0.13(2)$ & fixed & fixed \\
\hline $\mathrm{CH}_{3} \mathrm{OH} 2_{1}-1_{1} \mathrm{E}$ & $0.03(2)$ & $0.19(5)$ & fixed & fixed & $\lesssim 0.10$ & $\lesssim 0.03$ & & \\
\hline $\mathrm{CH}_{3} \mathrm{OH} 3_{0}-2_{0} \mathrm{~A}^{+}$ & $0.65(10)$ & $3.64(12)$ & $1.68(7)$ & $5.27(9)$ & $0.73(16)$ & $1.21(7)$ & $4.48(5)$ & $1.56(7)$ \\
\hline $\mathrm{CH}_{3} \mathrm{OH} 3_{-1}-2_{-1} \mathrm{E}$ & $0.60(10)$ & $3.38(12)$ & fixed & fixed & $0.51(16)$ & $0.84(8)$ & fixed & fixed \\
\hline $\mathrm{CH}_{3} \mathrm{OH} 3_{0}-2_{0} \mathrm{E}$ & $\lesssim 0.28$ & $\lesssim 0.30$ & & & & & & \\
\hline \multirow[t]{2}{*}{ CS 3-2 } & $0.91(14)$ & $1.10(14)$ & $4.88(5)$ & $1.15(11)$ & $1.6(2)$ & $2.20(12)$ & $4.58(3)$ & $1.28(2)$ \\
\hline & $0.78(14)$ & $4.5(3)$ & $1.88(15)$ & $5.4(3)$ & & & & \\
\hline SO $3_{2}-2_{1}$ & $0.35(5)$ & $2.21(8)$ & $1.99(10)$ & $5.7(2)$ & $0.67(7)$ & $0.88(4)$ & $4.56(3)$ & $1.22(7)$ \\
\hline $\mathrm{SO} 6_{5}-5_{4}{ }^{c}$ & $0.15(7)$ & $0.80(10)$ & $1.6(3)$ & $4.7(5)$ & $0.36(8)$ & $0.57(6)$ & $4.22(7)$ & $1.4(2)$ \\
\hline $\mathrm{SO} 7_{6}-6_{5}^{c}$ & $0.2(1)$ & $0.72(18)$ & $1.6(3)$ & $3.5(5)$ & $0.3(1)$ & $0.17(5)$ & $4.3(1)$ & $0.5(2)$ \\
\hline $\mathrm{H}_{2} \mathrm{~S} 1_{1,0}-0_{0,1}$ & $0.2(1)$ & $0.9(2)$ & $2.8(4)$ & $3.9(9)$ & $0.3(1)$ & $0.5(2)$ & $4.3(2)$ & $1.5(4)$ \\
\hline
\end{tabular}

${ }^{0}$ The ${ }^{13} \mathrm{CO} 1-0$ is fitted with three Gaussian components. One is forced to have the same velocity peak and line width as the $\mathrm{C}^{18} \mathrm{O}$ emission. ${ }^{a}$ At the ambient velocity $\left(\sim 4.8 \mathrm{~km} \mathrm{~s}^{-1}\right)$ the emission is missing. Computed the moments for the broader component using the CS velocity range $\left(-0.6-4.8 \mathrm{~km} \mathrm{~s}^{-1}\right)$.

${ }^{b}$ Used two components fits as in CS 3-2.

${ }^{d}$ Very marginal detection.

${ }^{c}$ Marginal detection, so we used the -2.2 to $5.2 \mathrm{~km} \mathrm{~s}^{-1}$ velocity range to derive the integrated line intensities, velocity and line width. 